\title{
COMPARISON OF A NINTENDO WII BALANCE BOARD WITH A LABORATORY-GRADE FORCE PLATE ON MEASUREMENT OF TRANSITIONAL MOVEMENTS
}

\author{
Francesco Sgròn ${ }^{1}$, Roberto Coppola ${ }^{2}$, Salvatore Pignato ${ }^{1}$, and Mario Lipoma ${ }^{1}$ \\ ${ }^{1}$ Faculty of Human and Society Sciences - University of Enna "Kore”, Enna, EN, Italy \\ ${ }^{2}$ University of Split, Faculty of Kinesiology, Split, Croatia
}

Original scientific paper

https://doi.org/ 10.26582/k.51.1.2

\begin{abstract}
:
The aim of this study was to verify whether the Nintendo Wii-Balance-Board was valid and reliable for assessing sit-to-stand and return-to-sit tasks by comparing it with a gold-standard force plate. Ten elderly (age $=78.21 \pm 14.82$ years; males $=4$; females $=6$ ) and eleven young $($ age $=24.25 \pm 12.43$ years; males $=6$; females $=5$ ) participants of both genders performed five sit-to-stand and return-to-sit tasks consecutively by placing their feet on the Wii-Balance-Board; after two days of rest, they repeated the same assessment. The Wii-BalanceBoard was positioned over the force plate to concurrently acquire the vertical component of ground reaction forces. Relevant kinetic and temporal parameters were estimated from these signals. Both the Wii-BalanceBoard and force plate measurements resulted in a high level of correlation for almost all the parameters (Pearson's product-moment $r$ ranged from 0.91 to $0.99, \mathrm{p}<.001$ ) and, for the same parameters, intra-class correlation coefficients revealed a high level of agreement between the devices (ranged from 0.93 to 0.99 ). Bland-Altman plots and regression analysis detected systematic and fixed biases for two parameters (i.e., the inclination of force in standing and rising), while other parameters resulted with none systematic biases; the absolute magnitude of those differences was trivial or small (standardized biases ranged from 0.01 to 0.4 ). A high level of intra-device reliability was measured for all the parameters (intra-class correlation coefficients ranged from 0.85 to 0.99 ). The Wii-Balance-Board proved valid and reliable in comparison with a force plate for assessing transition movements so it can be considered a valuable solution for supporting the assessment procedures of average practitioners.
\end{abstract}

Key words: sit-to-stand, return-to-sit, validity analysis, low-cost device, reliability analysis

\section{Introduction}

The assessment of abilities in performing transitional movements represents an essential research topic in the prevention of falls and reduction of injury risks (Whitney, et al., 2005). In this respect, sit-to-stand (STS) and return-to-sit (RTS) tasks are widely performed during several daily activities and their clinical assessment can be used for investigating the related motor strategies in the young and elderly (Papa \& Cappozzo, 2000). In detail, STS is used to assess residual strength in the elderly and explosive strength in young people, while RTS is used for evaluating specific kinetic parameters and eccentric force. Of note, eccentric force during aging does not decrease as much as does the explosive force in the lower limbs (Hortobágyi, et al., 1995; Roig, et al., 2010); the assessment of this functional index allows a holistic perspective of an individual's functional mobility status. In this respect, several clinical tests discussed in the literature (e.g., sit-to-stand, five-repetition sit-to-stand, short physical performance battery) have been criticized for weak validity, reliability, and accuracy level in relation to the functional status of the measured participants and to the assessors' level of ability and experience (Silva, Quintino, Franco, \& Faira, 2014).

The approaches used as an alternative to clinical tests are often performed by means of a laboratory-grade force-plate (FP). This device is identified as the gold standard for the assessment of the afore-mentioned tasks (Zijlstra, Mancini, Lindemann, Chiari, \& Zijlstra, 2012), but it is expensive, complex, and normally located in laboratory settings or clinical centres. These are significant drawbacks, and some of them (i.e., the need for a laboratory setting) may violate the affordance constraints required by an ecological task assessment (Davis \& Burton, 1991); furthermore, elderly 
participants may have trouble with accessing these sites. According to this scenario, the need for an alternative, low-cost, widely available, easy-to-use, and portable device is guaranteed.

The Nintendo Wii Balance Board (WBB) is an exergaming technology that appears to satisfy these demands. The WBB is a plate composed of four transducers that are used to acquire the forces provided above the board's surface. This device has already been compared with the gold standard force plates in terms of its usage feasibility and inter- and intra-device validity (i.e., in comparison with FPs and with the same WBB, respectively) for assessing postural control abilities (Clark, et al., 2010; Clark, Mentiplay, Pua, \& Bower, 2018; Huurnink, Fransz, Kingma, \& van Dieën, 2013; Merchant-Borna, et al., 2017; Pagnacco, Oggero, \& Wright, 2010; Park \& Lee, 2014; Pavan, Cardaioli, Ferri, Gobbi, \& Carraro, 2015; Sgrò, Monteleone, Pavone, \& Lipoma, 2014). Studies about intradevice reliability showed intra-class correlation coefficients ranging from poor to excellent, while inter-device validity was found excellent if the same posturography parameters were acquired from the plates. About the posturography parameters, the total length of the center of pressure $(\mathrm{CoP})$ trajectory and the relative mean velocity resulted with the highest validity and reliability in the double limb standing test. The validity of the WBB for assessing the oscillation of $\mathrm{CoP}$ have also been verified during squatting exercise (Mengarelli, et al., 2018). Moreover, weight-bearing asymmetry of the lower limbs in clinical populations has previously been studied by two WBBs concurrently and the validity of this approach was verified (Abujaber, Gillispie, Marmon, \& Zeni, 2015; Clark, Howells, Feller, Whitehead, \& Webster, 2014); also, validity of the WBB for measuring lower-limb strength performance has been examined and verified in healthy young population (Jorgensen, Andersen, Ryg, \& Masud, 2015; Yamamoto \& Matsuzawa, 2013). Recently, data related to ground reaction force and CoP position acquired from a WBB during STS task have been used to estimate novel indexes for quantitative assessment of this motion and, then, they have been discussed in relation to the times of timed up and go (TUG) test (Yamako, Chosa, Totoribe, Fukao, \& Deng, 2017). Results showed the significant differences between the novel indexes and the TUG times among the participants' agegroups, but inter-device (i.e., in comparison with a gold standard force plate) and intra-device validity analyses of these indexes provided good results. However, the available literature on the use of WBB for assessing kinetic parameters during dynamic task is limited and, to the best of the authors' knowledge, there is still a need for a comparison of this device with a FP for the biomechanical assessment of STS and RTS in a practical setting. Hence, the purpose of this study was to evaluate the WBB as a standalone device for its utility in assessing the transitional movements required in STS and RTS tasks by comparing it with a FP. We hypothesized the following:

H1: The parameters obtained by means of the WBB will be in a significant and positive agreement with those obtained by the simultaneous use of a gold-standard force plate.

H2: The parameters obtained by the WBB will show strong intra-device reliability for the participant assessment.

\section{Method}

\section{Participants and procedures}

We recruited 10 community-dwelling older adults, 4 males and 6 females, with the following characteristics: age $=78.21 \pm 14.82$ years; body mass: $55.99 \pm 11.89 \mathrm{~kg}$; body height $=1.50 \pm 0.07 \mathrm{~m}$. We also recruited 11 healthy young subjects, 6 males and 5 females, with the following characteristics: age $24.25 \pm 12.43$ years; body mass: $60.19 \pm 11.34$ $\mathrm{kg}$; body height $=1.67 \pm 0.07 \mathrm{~m}$. Participants were included if they were free of physical impairments and were able to perform transitional movements without any additional aid. Elderly were not involved in any adapted physical activity program, while young participants performed self-selected free play. The participants performed STS and RTS tasks without the help of their arms because their palms were crossed over their chest. According to previous studies (Guralnik, et al., 1994; Janssen, Bussmann, \& Stam, 2002; Zijlstra, et al., 2012), we used a chair (seat height: $0.47 \mathrm{~m}$, seat depth: 0.45 $\mathrm{m})$ without a back or arms, and a wedge was placed under that chair to line up the participants' feet with the WBB's height (see Figure 1).

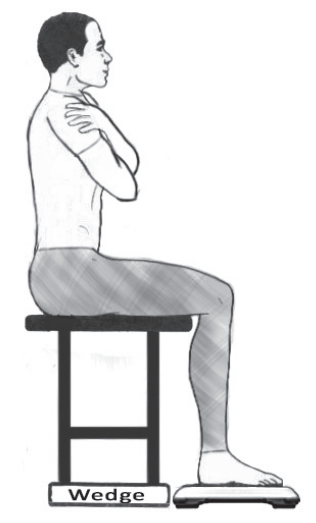

Figure 1. The subjects performed STS and RTS by placing his/ her feet on the $W B B$.

Two-foot silhouette, equally spaced from the WBB center, were applied above the plate to provide a standardized foot placement and to ensure the intra-device reliability too. 
The assessment procedures were accomplished on two different days in a research laboratory where two technicians, with two years of expertise in biomechanical assessment of human movement, waited for the participants and showed them the procedures. Each assessment session was scheduled at the same time in the morning, two hours after the participants have finished their breakfast. Before the assessment, each participant was familiarized with the environment and the assigned tasks by performing two trials. Then, the participants performed the sit-to-stand followed by the return-to-sit movement for five times as quickly and safely as they could. A technician was next to the participants during the assessment procedures to ensure their safety.

The participants involved in this research gave their consent before the assessment procedure and the study was carried out in accordance with the Declaration of Helsinki. The Ethical Committee of the University of Enna and the Ethical Board of the nursing home approved the current study.

\section{Data acquisition and analysis}

The WBB is a device normally used for entertainment applications and it was positioned above a gold standard force plate (AMTI, Model OR6-7, Watertown, MA, USA) to acquire the vertical component of ground reaction force (vGRF) signals simultaneously by avoiding within-subject variability. The weight of the WBB was removed from the AMTI signal.

Two computers (PCs) were used for the data acquisition: one $\mathrm{PC}$ was connected to the WBB using a Bluetooth interface and the other was connected to the AMTI by means of an analogueto-digital converter. The data from the WBB were acquired using an ad-hoc application developed with C\# and XNA Library, while the data from the AMTI were acquired by means of Vicon Nexus software (Vicon, ver. 2.0, Oxford Metrics, Oxford, UK). The output rate was $50 \mathrm{~Hz}$ for the WBB and $1000 \mathrm{~Hz}$ for the AMTI. The calibration of the WBB sensors was done by applying multiple known loads over the plate in the testing position and estimating offset factors for each sensor (Clark, et al., 2014). Then, the vGRF was calculated by summing the values of each calibrated sensor. AMTI was calibrated in accordance with the manufacturer's recommendations.

Using ad-hoc script built in a Matlab environment (The Mathworks, Natick, RI, USA), the vGRF signals of the WBB and AMTI, respectively, were processed to obtain the indicators that described the participants' performance in the aforementioned tasks. Those signals were initially filtered by means of a Butterworth low-pass filter. Using residual analysis (Winter, 1995), the cut-off frequencies were estimated to $12 \mathrm{~Hz}$ and $10 \mathrm{~Hz}$ for the WBB and AMTI, respectively. Then, the signals of the five trials were processed for the estimation of the parameters described in Table 1. Several of those parameters were in agreement with the ones provided in previous studies (Lindemann, et al., 2003; Sgrò, Licari, Coppola, \& Lipoma, 2015; Zijlstra, et al., 2012) and were according to the events identified by Kralj, Jaeger and Munih (1990). A vGRF signal with the indication of the relevant events of the two tasks is shown in Figure 2.

Table 1. Temporal events, temporal phases, and kinetic parameters identified from the vertical component of the ground reaction force signal acquired during the sit-to-stand (STS) and return-to-sit (RTS) tasks

\begin{tabular}{|c|c|c|c|}
\hline Task & Variables & Code & Description \\
\hline \multirow{10}{*}{ STS } & $\begin{array}{l}\text { Start of standing phase } \\
\text { [frame] }\end{array}$ & T1 & The first point when the vGRF decreases more than $2.5 \%$ of the feet weight. \\
\hline & $\begin{array}{l}\text { Instant of max vGRF } \\
\text { [frame] }\end{array}$ & $\mathrm{T} 2$ & The point when the vGRF reaches its maximum value during the standing. \\
\hline & $\begin{array}{l}\text { Extension of the body } \\
\text { [frame] }\end{array}$ & T3 & $\begin{array}{l}\text { The first point when the vGRF reaches the body mass after the decreasing } \\
\text { and the increasing phase. }\end{array}$ \\
\hline & $\begin{array}{l}\text { The end of STS task } \\
\text { [frame] }\end{array}$ & T4 & The first point when the vGRF oscillates around $\pm 2.5 \%$ of the body weight. \\
\hline & Preparation phase $[\mathrm{s}]$ & P1 & The time between the points $\mathrm{T} 1$ and $\mathrm{T} 2$. \\
\hline & Rising phase $[\mathrm{s}]$ & $\mathrm{P} 2$ & The time between the points $\mathrm{T} 2$ and $\mathrm{T} 3$ \\
\hline & Stabilization phase $[\mathrm{s}]$ & P3 & The time between the point T3 and the end of the sit-to-stand task (T4). \\
\hline & $\begin{array}{l}\text { Normalized max. vGRF } \\
{[\mathrm{N} / \mathrm{kg}]}\end{array}$ & $\max \_v G R F_{N}$ & The ratio between the vGRF in T2 and the participant's body weight. \\
\hline & Overshoot $[\mathrm{N}]$ & OS & $\begin{array}{l}\text { The difference between the maximum value of the vGRF and the participant's } \\
\text { body weight. }\end{array}$ \\
\hline & Incline & IC_Stand & $\begin{array}{l}\text { The slope of the vGRF curve from the } 20 \% \text { to } 90 \% \text { of the signal estimated } \\
\text { between the points T1 and T2. }\end{array}$ \\
\hline
\end{tabular}




\begin{tabular}{|c|c|c|c|}
\hline Task & Variables & Code & Description \\
\hline \multirow{7}{*}{ RTS } & $\begin{array}{l}\text { Start of sitting phase } \\
\text { [frame] }\end{array}$ & T5 & $\begin{array}{l}\text { The first point when the vGRF decreases more than } 1.5 \% \text { of the body weight } \\
\text { after the stabilization phase (P3). }\end{array}$ \\
\hline & $\begin{array}{l}\text { Instant of max vGRF } \\
\text { [frame] }\end{array}$ & T6 & $\begin{array}{l}\text { The point when the vGRF reaches its maximum value after the start of the } \\
\text { sitting phase. }\end{array}$ \\
\hline & $\begin{array}{l}\text { Instant of min vGRF } \\
\text { [frame] }\end{array}$ & $\mathrm{T7}$ & $\begin{array}{l}\text { The point where the vGRF reaches its minimum value after the start of the } \\
\text { sitting phase. }\end{array}$ \\
\hline & Preparation phase $[\mathrm{s}]$ & P4 & The time between the points $\mathrm{T} 5$ and $\mathrm{T} 6$. \\
\hline & Sitting phase $[\mathrm{s}]$ & P5 & The time between the points $\mathrm{T} 6$ and $\mathrm{T} 7$. \\
\hline & $\begin{array}{l}\text { Normalized Eccentric } \\
\text { Force }[\mathrm{N} / \mathrm{kg}]\end{array}$ & $\mathrm{EF}_{\mathrm{N}}$ & The ratio between the vGRF in T6 and the participant's body weight. \\
\hline & Incline & IC_Sit & $\begin{array}{l}\text { The slope of the vGRF curve from the } 20 \% \text { to } 90 \% \text { of the signal estimated } \\
\text { between the points T6 and T7. }\end{array}$ \\
\hline
\end{tabular}

Note. STS: sit-to-stand; RTS: return-to-sit; vGRF: vertical component of ground reaction force; s: seconds; N: newton; kg: kilogram.

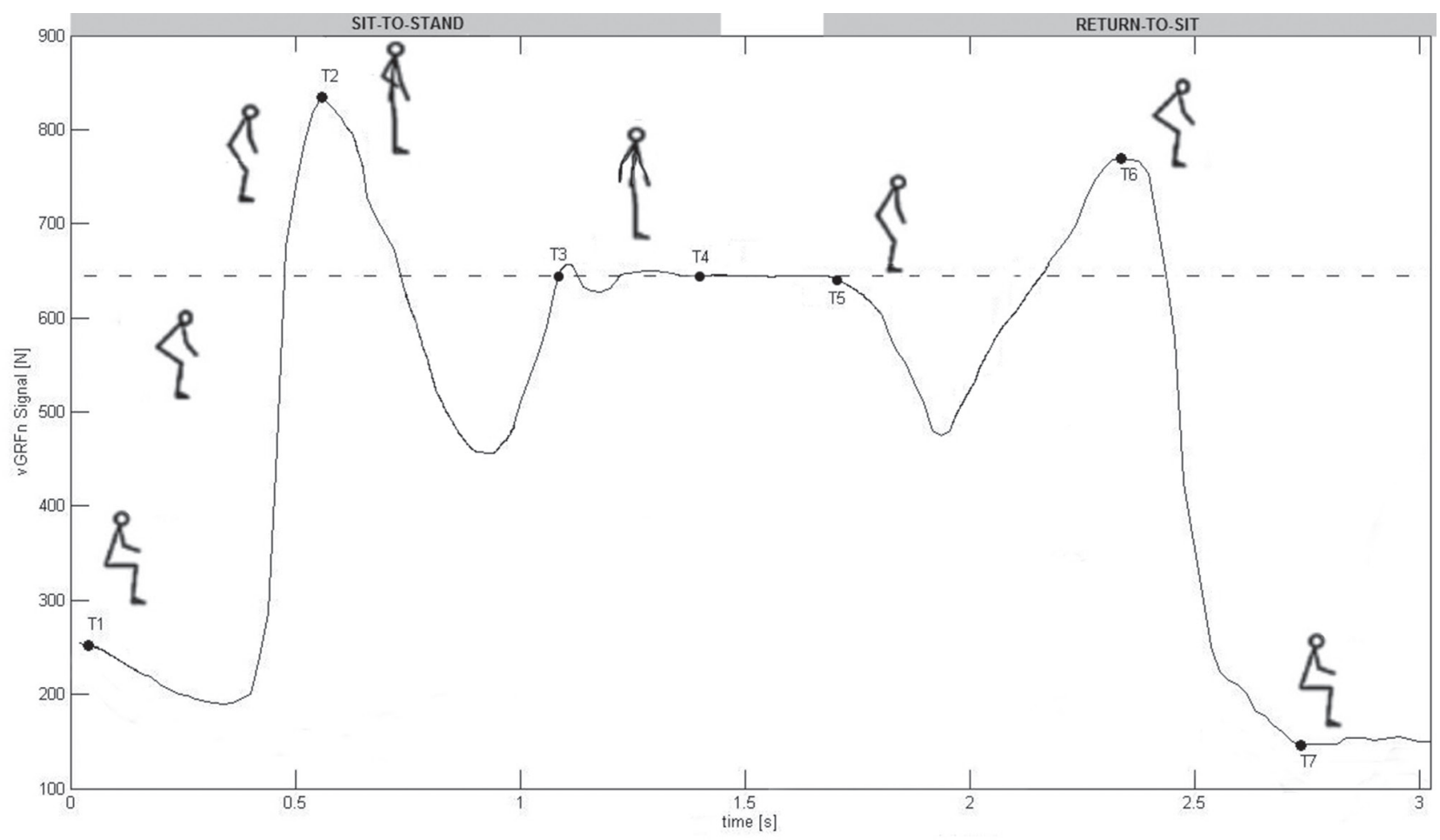

Figure 2. The vertical component of ground reaction force signal ( $v G R F)$ acquired from the Wii Balance Board. The continuous line represents the $v G R F$, while the dotted line represents body weight of a participant. The black circles in the vGRF identify the following frames: the start of the standing phase (T1), the instant of the maximum vGRF after the start of the standing movement (T2), the frame when the $v G R F$ reaches the body mass value (T3), the start of a stable posture at the end of the standing phase (T4), the start of the sitting phase (T5), the instant of the maximum vGRF during the sitting phase (T6), and the end of the sitting phase (T7). In the plot there are also several stick-figures for identifying the postures during the sit-to-stand and return-to-sit tasks, respectively.

\section{Statistical analysis}

Data were preliminary checked for accuracy, missing values, outliers, and normal distribution (using Kolmogorov-Smirnov test), and then statistical analyses were performed in three steps. First, the Wii Balance Board's concurrent validity was tested using Pearson's product-moment correlation coefficient $(r)$ via bootstrapping $(\mathrm{n}=1,000)$. Second, inter-device reliability was analysed by intra-class correlation coefficient (ICC) with $90 \%$ CI. The level of agreement was measured with
Bland-Altman (BA) plots and reported via mean differences, $95 \%$ limits of agreement and the analysis of the regression line on BA plots (Giavarina, 2015). Moreover, the presence of biases was verified with the following procedures: the proportional biases were detected if the slope of the regression line applied to the data of Bland-Altman plots was statistically different from 0 , while the fixed biases were estimated by testing if the mean differences were statistically different from 0 on the basis of the one-sample $t$-test (Ludbrook, 2002). Finally, the 
standardised biases were also quantified by using a specifically-designed spreadsheet proposed by Hopkins (2000).

Third, the intra-device reliability of the WBB was estimated by ICC values and the examination of the standard error of measurement (SEM), which was calculated using the following formula: $\mathrm{SEM}=\mathrm{SD} *[\sqrt{ }(1-\mathrm{ICC})]$, where $\mathrm{SD}$ was the standard deviation of the test and ICC was the reliability coefficient.

The values of the ICC were interpreted as poor (0.00-0.39), modest (0.40-0.74), and excellent (0.75-1) (Fleiss, 2011). The values of the standardised bias were interpreted as trivial $(<0.2)$, poor (0.2-0.6), moderate (0.6-1.2), large (1.2-2.0), and very large (>2.0) (Hopkins, 2000). The statistical analysis was conducted by SPSS v. 21.0 (SPSS Inc. Chicago, IL, USA) and a specifically-designed spreadsheet (Hopkins, 2000). The level of significance was set to $\mathrm{p} \leq .05$.

\section{Results}

One participant missed the second assessment, so he was not considered in further analysis. The preliminary check did not identify any missing values or outliers. Furthermore, all the assumptions required for the intra-class correlation and regression analysis were verified and met.

\section{Concurrent validity of the WBB}

Table 2 shows the statistics of the WBB concurrent validity for the young and elderly, separately.

Table 2. Statistics of concurrent validity analysis between the Wii Balance Boards and force plate (AMTI) in the assessment of sit-to-stand and return-to-sit tasks for the young and the elderly

\begin{tabular}{|c|c|c|c|c|c|c|}
\hline \multirow[b]{2}{*}{ Parameter } & \multirow[b]{2}{*}{ Group } & \multicolumn{2}{|c|}{ Correlation analysis } & \multicolumn{3}{|c|}{ Regression analysis } \\
\hline & & r & $90 \% \mathrm{Cl}$ & slope & $\mathrm{R}^{2}$ & $p$ \\
\hline \multirow[t]{2}{*}{$\mathrm{P} 1[\mathrm{~s}]$} & Young & $0.91^{* *}$ & $0.86,0.97$ & 0.84 & 0.83 & $<.0001$ \\
\hline & Elderly & $0.94^{* *}$ & $0.89,0.98$ & 0.71 & 0.89 & $<.0001$ \\
\hline \multirow[t]{2}{*}{$\mathrm{P} 2$ [s] } & Young & $0.94^{* *}$ & $0.85,0.98$ & 1.07 & 0.88 & $<.0001$ \\
\hline & Elderly & $0.90^{* *}$ & $0.80,0.96$ & 0.84 & 0.81 & $<.0001$ \\
\hline \multirow[t]{2}{*}{ P3 [s] } & Young & $0.99^{* *}$ & $0.99,1.00$ & 0.97 & 0.99 & $<.0001$ \\
\hline & Elderly & $0.96^{\star *}$ & $0.91,0.99$ & 0.93 & 0.99 & $<.0001$ \\
\hline \multirow[t]{2}{*}{$\max \_v G R F_{N}[N / k g]$} & Young & $0.97^{* *}$ & $0.94,0.99$ & 0.99 & 0.95 & $<.0001$ \\
\hline & Elderly & $0.95^{\star *}$ & $0.43,0.99$ & 0.96 & 0.89 & $<.0001$ \\
\hline \multirow[t]{2}{*}{ OS [N] } & Young & $0.99^{* *}$ & $0.98,0.99$ & 0.96 & 0.98 & $<.0001$ \\
\hline & Elderly & $0.98^{\star *}$ & $0.91,0.99$ & 0.92 & 0.96 & $<.0001$ \\
\hline \multirow[t]{2}{*}{ IC_Stand } & Young & -0.76 & $-0.92,-0.48$ & -0.03 & 0.57 & 0.007 \\
\hline & Elderly & -0.53 & $-0.89,-0.15$ & -0.07 & 0.27 & 0.118 \\
\hline \multirow[t]{2}{*}{ P4 [s] } & Young & $0.96^{* *}$ & $0.90,0.99$ & 0.95 & 0.91 & $<.0001$ \\
\hline & Elderly & $0.93^{\star *}$ & $0.87,0.98$ & 0.72 & 0.86 & $<.0001$ \\
\hline \multirow[t]{2}{*}{ P5 [s] } & Young & $0.95^{\star *}$ & $0.86,0.99$ & 0.95 & 0.90 & $<.0001$ \\
\hline & Elderly & $0.87^{* *}$ & $0.64,0.99$ & 0.86 & 0.75 & 0.001 \\
\hline \multirow[t]{2}{*}{$E F_{N}[N / k g]$} & Young & $0.99^{* *}$ & $0.97,0.99$ & 1.04 & 0.98 & $<.0001$ \\
\hline & Elderly & $0.97^{\star \star}$ & $0.95,0.99$ & 1.23 & 0.93 & $<.0001$ \\
\hline \multirow[t]{2}{*}{ IC_Sit } & Young & -0.35 & $-0.82,0.18$ & -2.427 & 0.12 & 0.297 \\
\hline & Elderly & 0.38 & $-0.25,0.93$ & 1.904 & 0.14 & 0.273 \\
\hline
\end{tabular}

Note. r: Pearson's product-moment correlation coefficient; $90 \% \mathrm{Cl}$ : $90 \%$ confidence interval; $\mathrm{R}^{2}$ : r-squared or coefficient of determination; p: significant value; P1: preparation phase; P2: rising phase; P3: stabilization phase; max_vGRF ${ }_{N}$ : maximum value of the vertical component of ground reaction force (vGRF) normalized by the body mass; OS: overshoot; IC_Stand: slope of the vGRF during the standing phase; P4: preparation phase; P5: sitting phase; $\mathrm{EF}_{\mathrm{N}}$ : eccentric force measured as the maximum value of the vGRF normalized by the body mass; IC_Sit: slope of the vGRF during the sitting phase; ${ }^{* *}: p<.01$. 
The Pearson's product-moment correlation coefficients showed significant and very high relationships for almost all the parameters in both groups. Only the relationship for the parameters IC Stand and IC_Sit was not statistically significant.
The results of the regression analysis confirmed the high level of relationships between the two devices (see Figures $3 \mathrm{a}-3 \mathrm{~b}$ for the young and $4 \mathrm{a}-4 \mathrm{~b}$ for the elderly for more details).
(A)

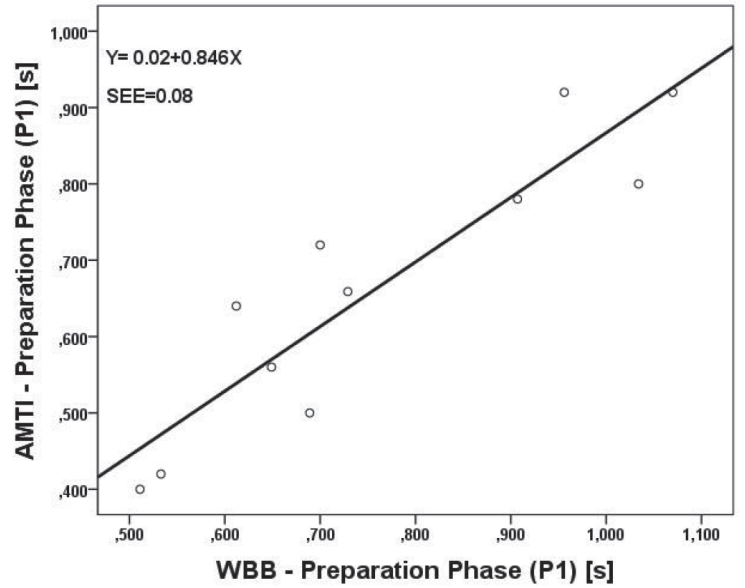

(C)

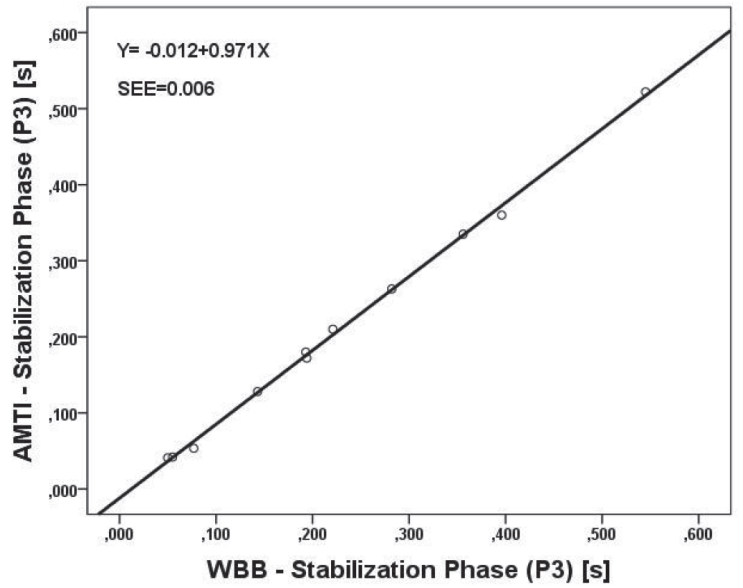

(E)

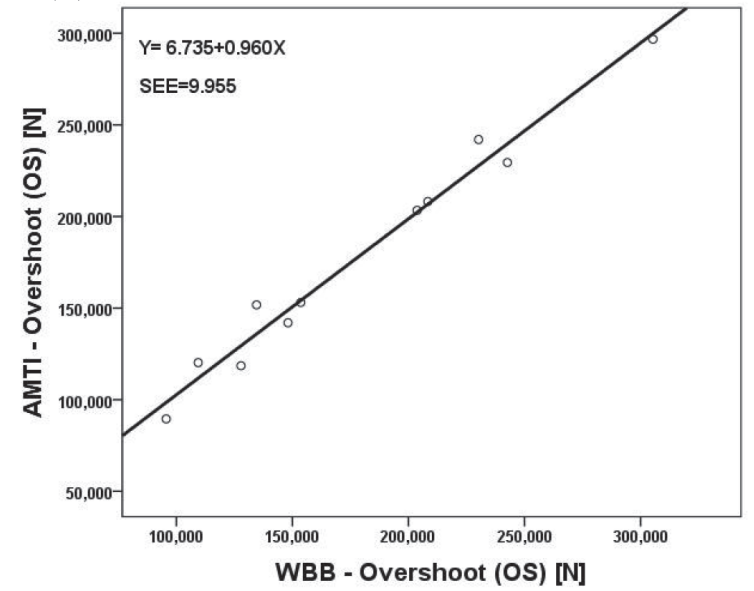

(B)

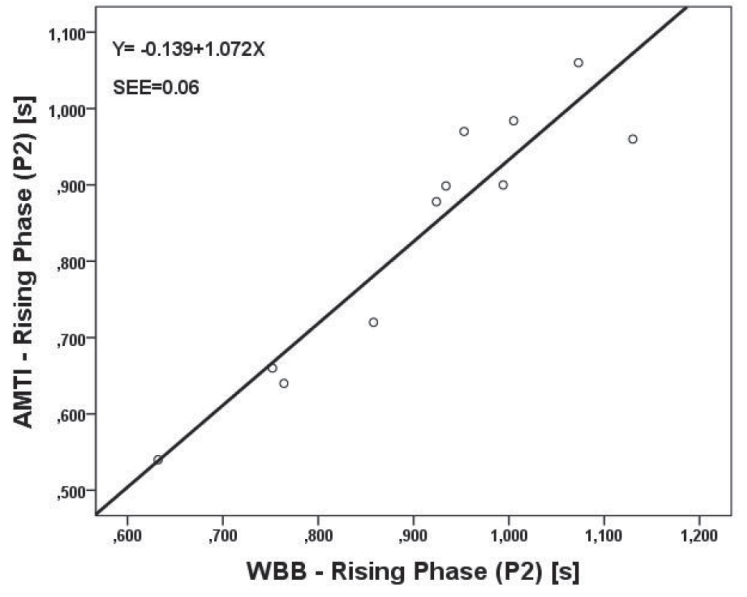

(D)

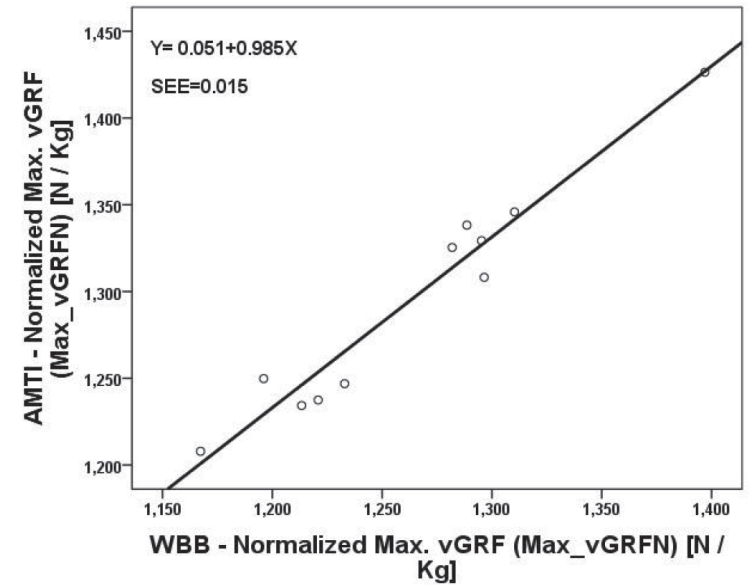

(F)

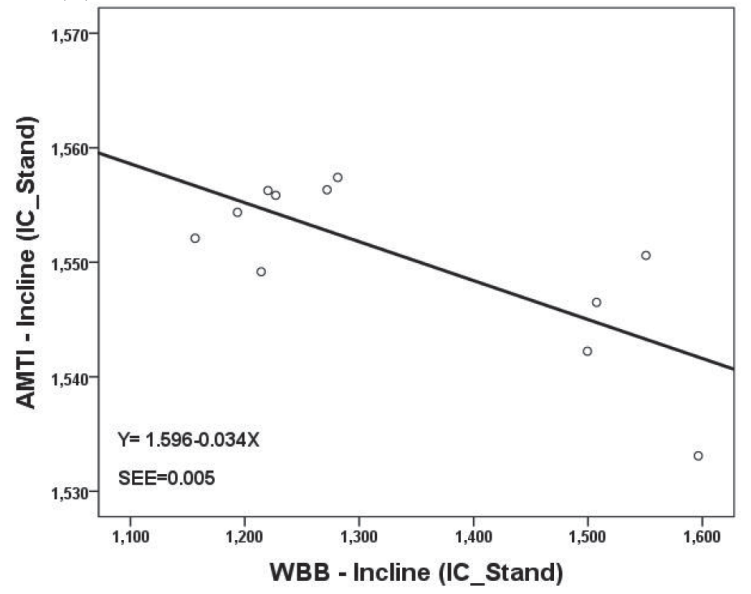

Figure $3 a$. Scatter plots representing the concurrent validity between the Wii Balance Board (WBB) and the laboratory-grade force platform (AMTI) for the parameters related to the STS task in the young group: (A) preparation phase; (B) rising phase; (C) stabilization phase; (D) normalized max vGRF; (E) overshoot; $(F)$ inclination of $v G R F$ in STS. Note. STS: sit-to-stand; SEE: standard error of estimates; max vGRF: maximum value of the vertical component of ground reaction force. 
(G)

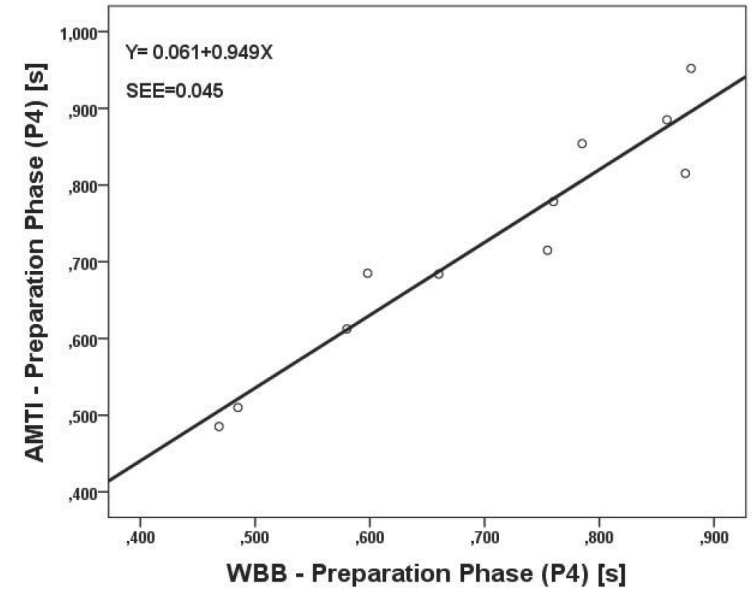

(I)

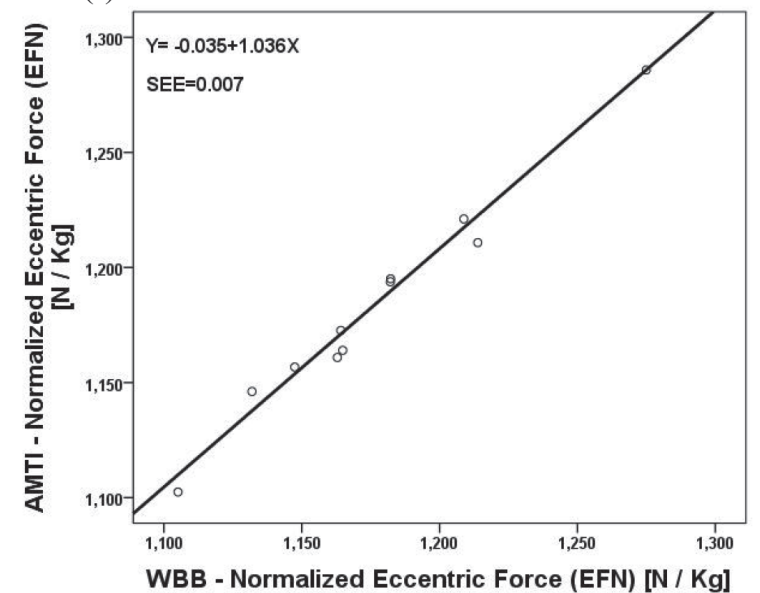

(H)

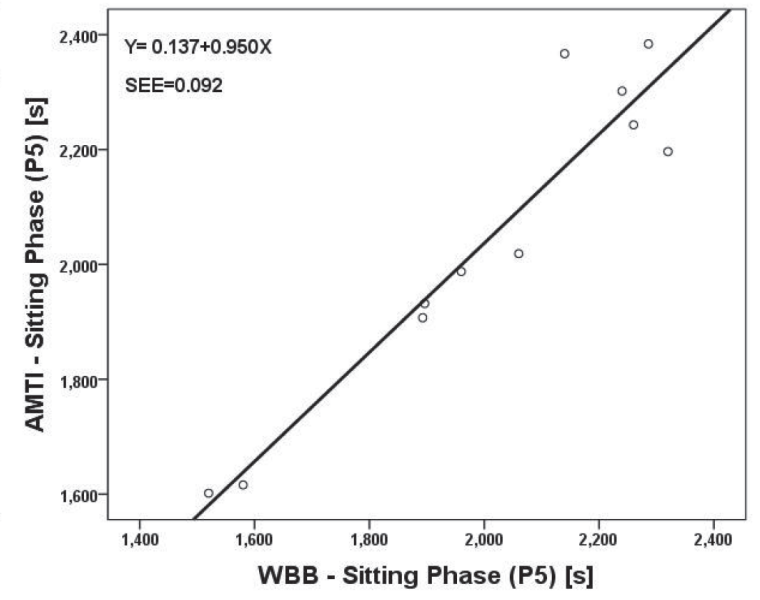

(L)

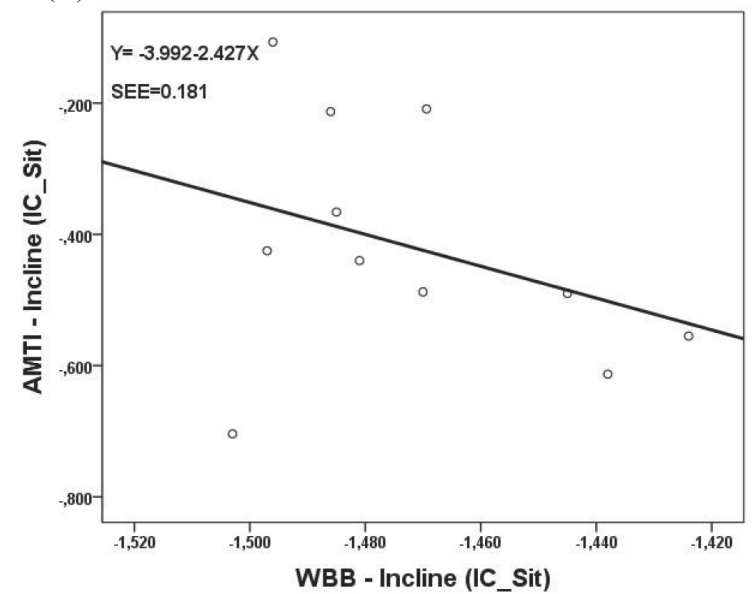

Figure 3b. Scatter plots representing the concurrent validity between the Wii Balance Board (WBB) and the laboratory-grade force platform (AMTI) for the parameters related to the RTS task in the young group: $(G)$ preparation phase; (H) sitting phase; (I) normalized eccentric force; (L) inclination of $v$ GRF in RTS. Note. RTS: return-to-sit; SEE: standard error of estimates; $v G R F$ : vertical component of ground reaction force.

(A)

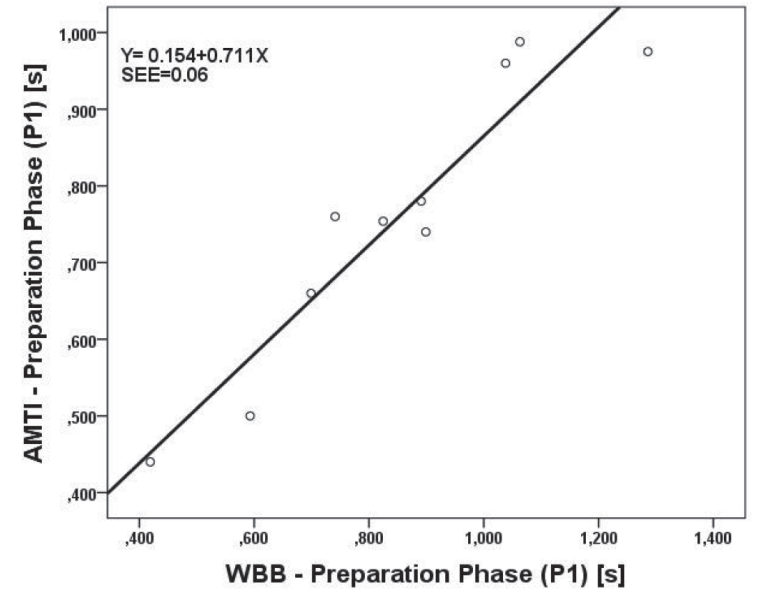

(B)

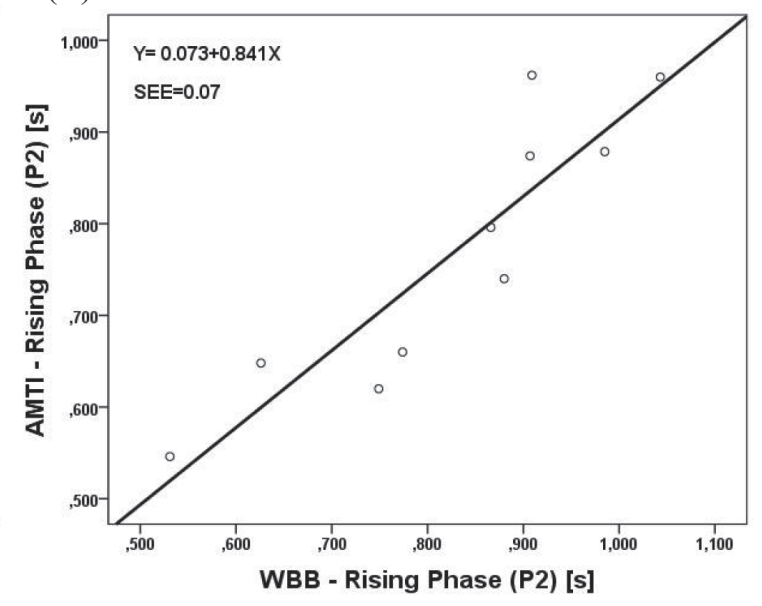


(C)

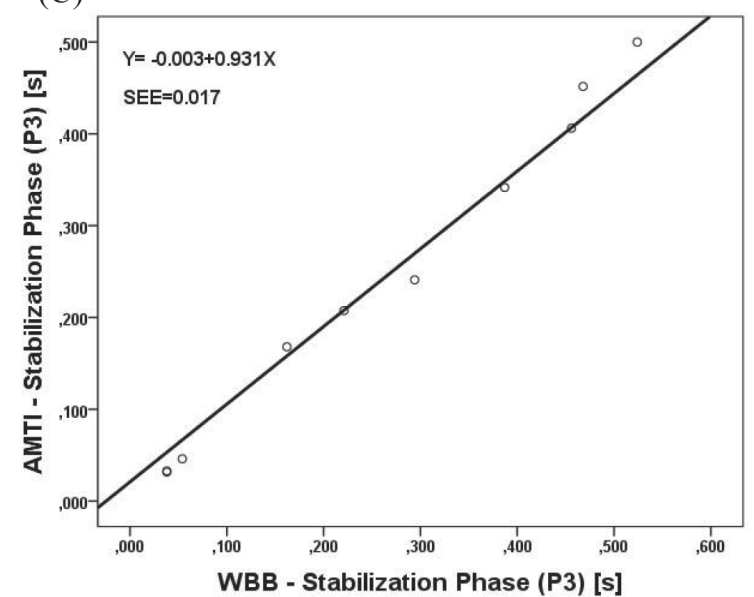

(E)

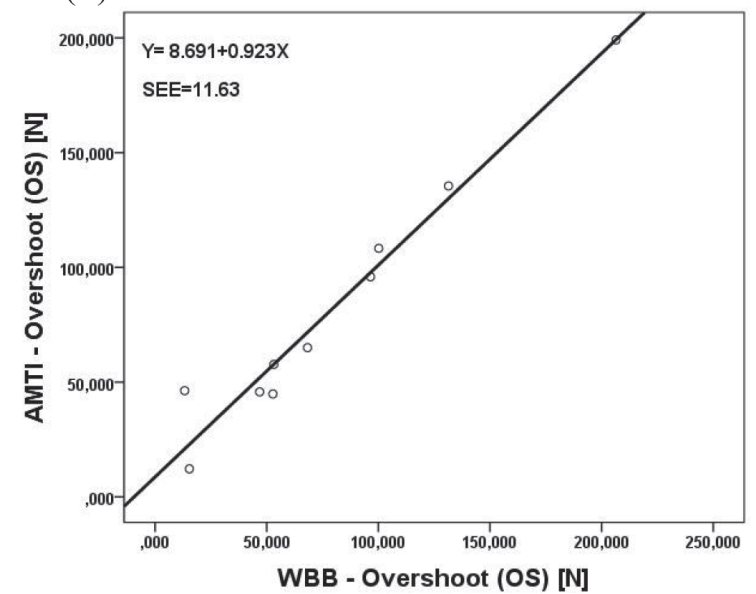

(D)

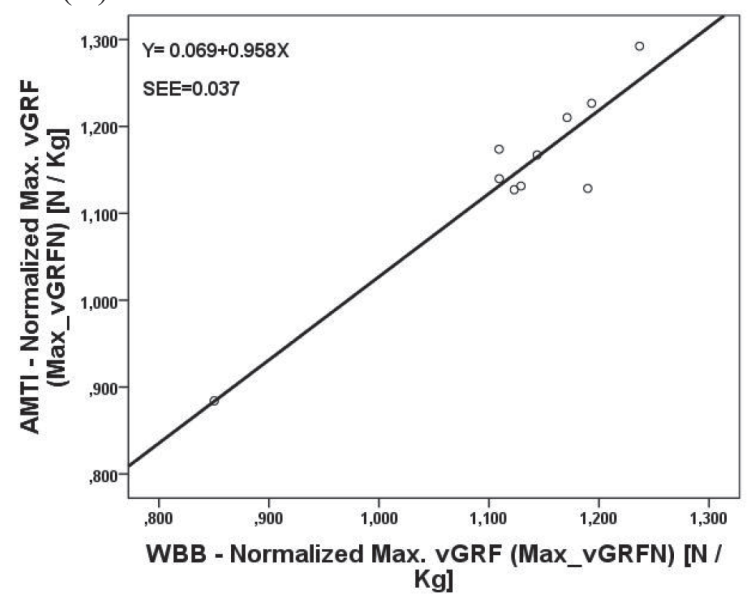

(F)

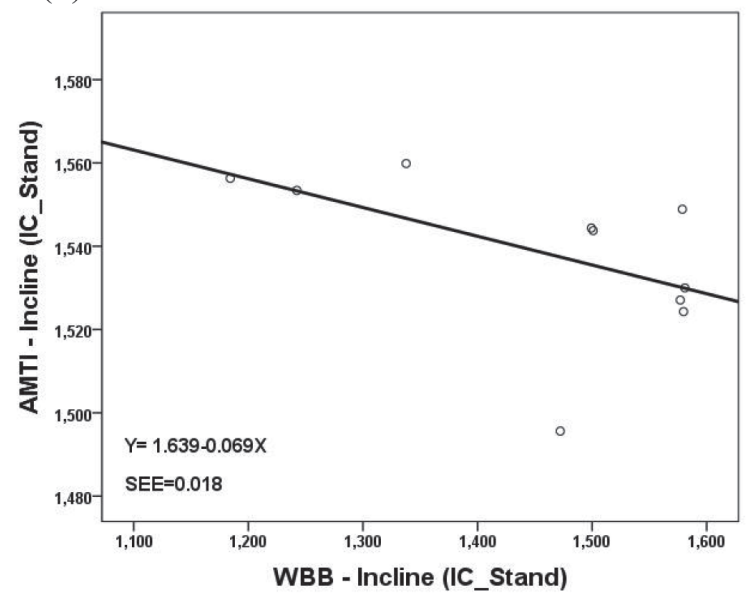

Figure 4a. Scatter plots illustrating the concurrent validity between the Wii Balance Board (WBB) and the laboratory-grade force platform (AMTI) for the parameters related to the STS task in the elderly group: (A) preparation phase; (B) rising phase; (C) stabilization phase; (D) normalized max vGRF; (E) overshoot; $(F)$ inclination of vGRF in STS. STS: sit-to-stand; SEE: standard error of estimates; max $v G R F$ : maximum value of the vertical component of ground reaction force.

(G)

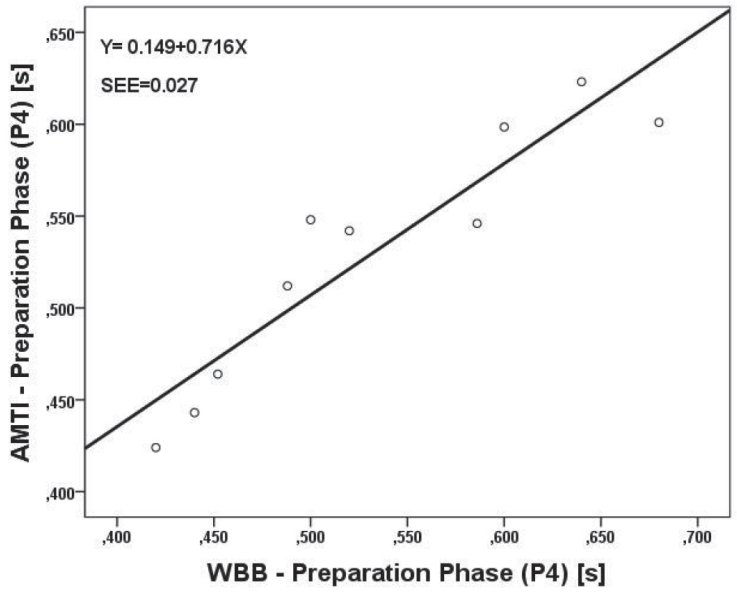

$(\mathrm{H})$

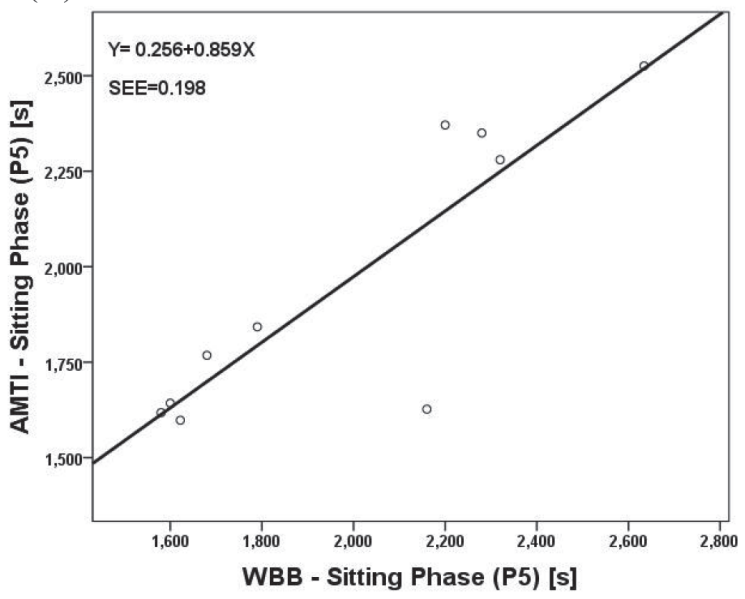


(I)

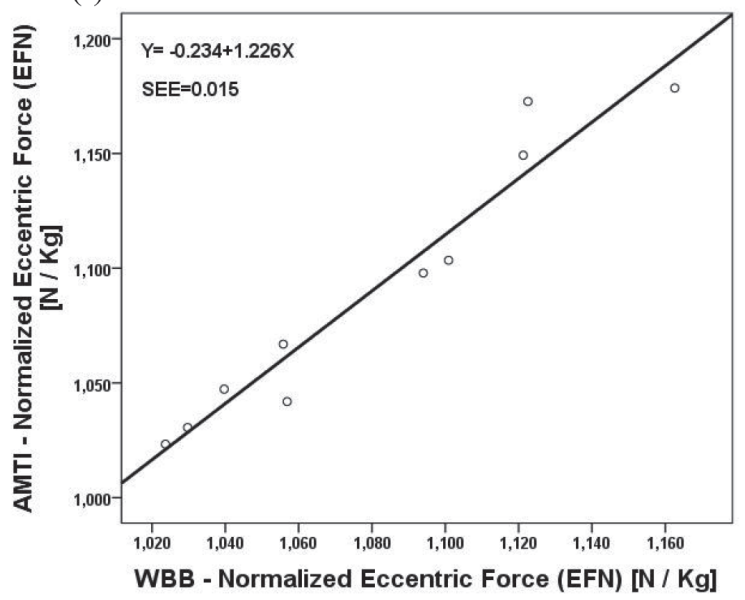

(L)

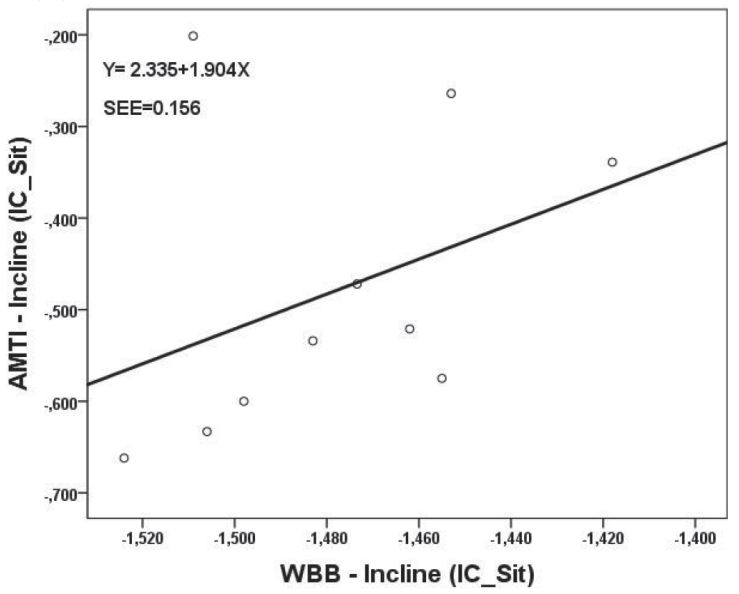

Figure 4b. Scatter plots illustrating the concurrent validity between the Wii Balance Board (WBB) and the laboratory-grade force platform (AMTI) for the parameters related to the RTS task in the elderly group: $(G)$ preparation phase; (H) sitting phase; (I) normalized eccentric force; (L) inclination of $v G R F$ in RTS. Note. RTS: return-to-sit; SEE: standard error of estimates; vGRF: vertical component of ground reaction force.

Notable, the statistics (i.e., slope and $\mathrm{R}^{2}$ ) for young group seem to be more in agreement with the predicted scores than the ones of elderly group.

\section{Reliability and accuracy of the measurement with the WBB versus the AMTI}

Table 3 shows the results of agreement and accuracy analyses for the measurements acquired by the WBB versus those concurrently measured by AMTI.

The ICCs revealed a very high level of agreement (ICCs ranged from 0.93 to 0.99 ) between almost all the parameters used for assessing the movement of STS and RTS in both groups. The level of agreement for IC stand and IC sit resulted not statistically significant. As regards accuracy analyses, the results did not reveal any proportional biases for the parameters except for the IC_stand and IC_sit in both groups (see Figures $5 \mathrm{a}-5 \mathrm{~b}$ for the young and $6 \mathrm{a}-6 \mathrm{~b}$ for the elderly, for detailed BlandAltman plots).

The small fixed bias was measured for standing phases (i.e., P1, P2 and P3) in both groups, and for kinetic parameters (i.e., $\max \mathrm{vGRF}_{\mathrm{N}}$ and $\mathrm{EF}_{\mathrm{N}}$ ) in the young. The absolute magnitude of the standardised biases were: a) trivial for overshoot, normalized eccentric force, stabilization, preparation (i.e., P4), and sitting phases, respectively; b) small for preparation phases (i.e., P1), rising phases and normalized maximum vGRF; c) very large for standing and sitting inclinations.

\section{Intra-device reliability}

The results of reliability analysis of the WBB measurements are shown in Table 4.
Excellent (ICC $>0.75)$ and significant correlations were measured for all parameters in both groups, whereas the values of SEM were limited.

\section{Discussion and conclusions}

Emerging, portable, inexpensive, and valid system for the quantitative assessment of human movement could represent a worthy resource for clinician and researchers. Previous studies have addressed validity and reliability of the Wii Balance Board (WBB) for assessing the kinetic characteristics of lower-limb movements (Abujaber, et al., 2015; Yamamoto \& Matsuzawa, 2013) and the standing balance (Clark, et al., 2010, 2017; Pagnacco, et al., 2010; Park \& Lee, 2014; Pavan, et al., 2015; Sañudo, Rueda, del Pozo-Cruz, de Hoyo, \& Carrasco, 2016; Sgrò, et al., 2014) in comparison with the goldstandard force plate (FP). Almost all studies found the WBB to be valid for the afore-mentioned aims but recommended to pay more attention to use WBB and FP interchangeably. Following a biomechanically oriented approach, the current study examined the concurrent validity as well as interand intra-device reliability of WBB to assess transition movements (i.e., sit-to-stand and return-to-sit) in comparison with the gold-standard force plate (i.e., AMTI), therefore the current results could contribute to extending the knowledge about the use of this device for quantitative assessment of human movement. Furthermore, we extend the current evidences on the use of WBB in the sit-tostand test (Yamako, et al., 2017) by providing indications about the accuracy of kinetic and temporal phases measurements related to this task and, also, related to return-to-sit task. Finally, although the use of two WBBs has been suggested and verified 


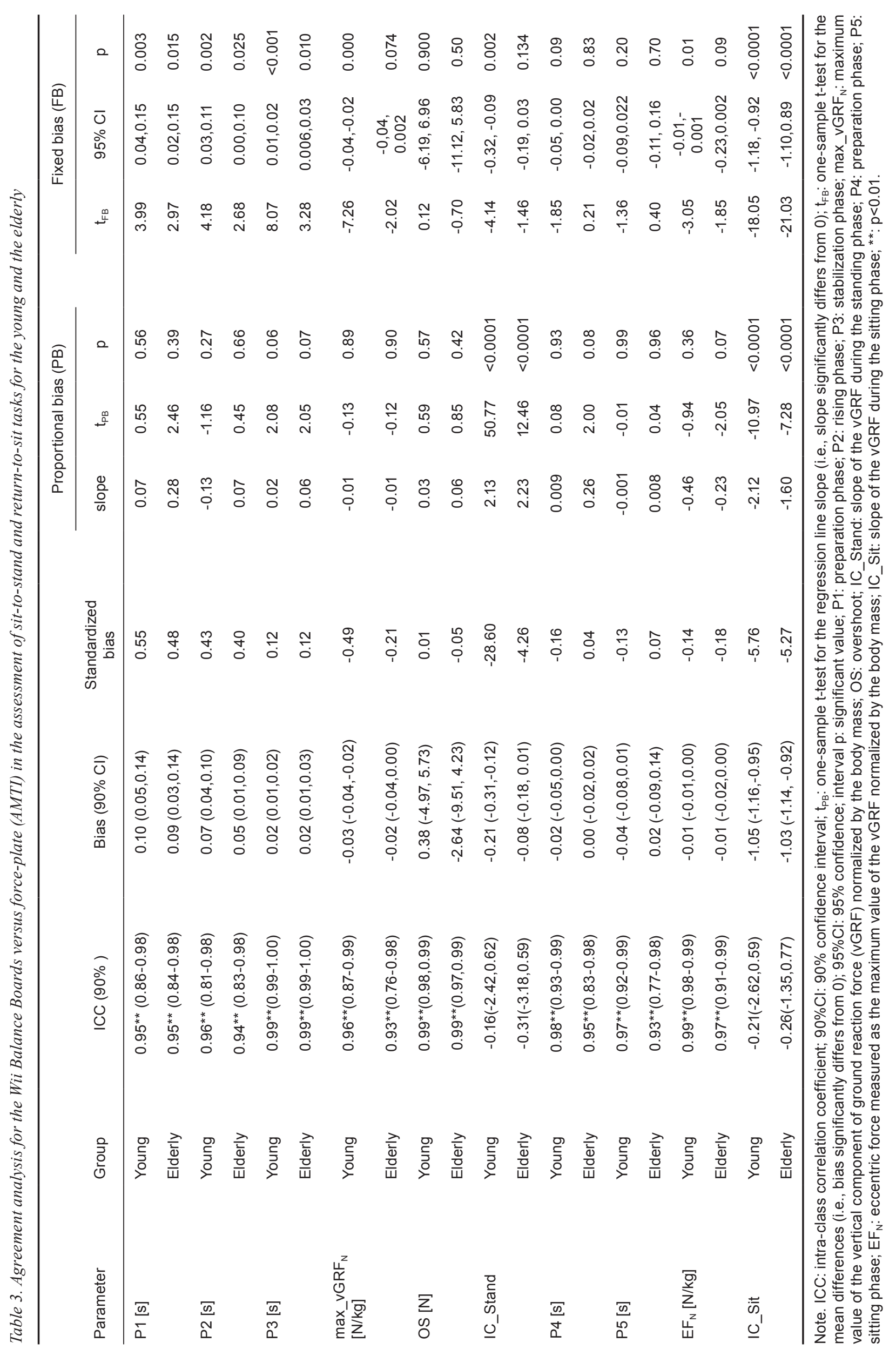


(A)

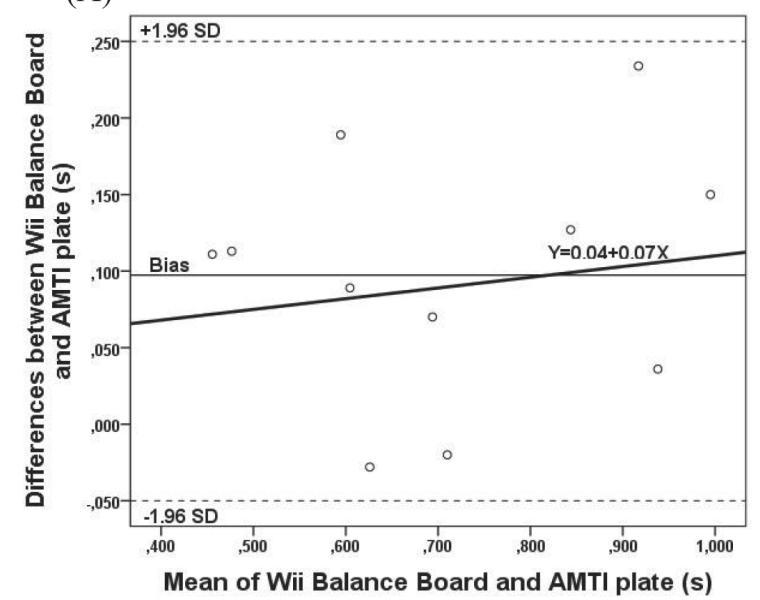

(C)

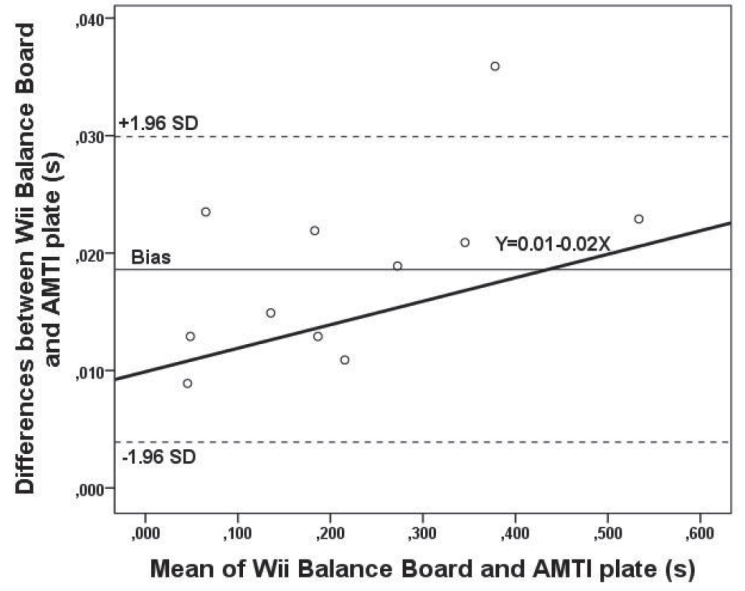

(E)

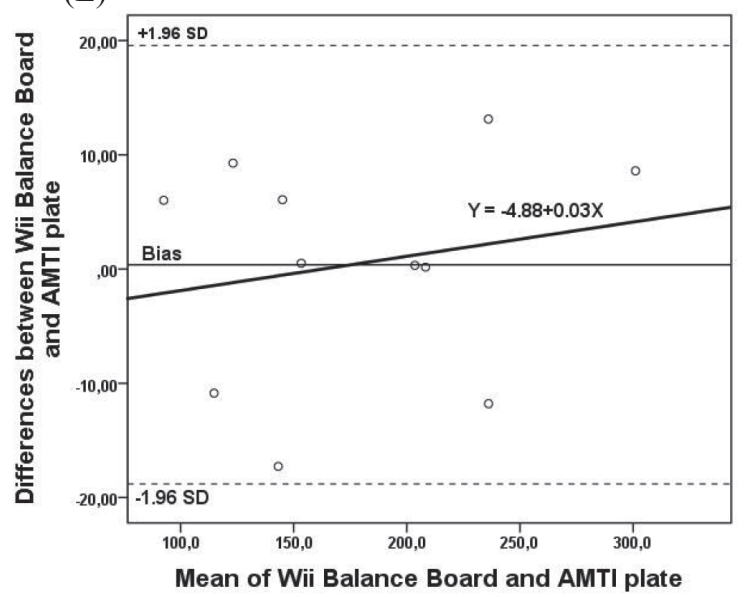

(B)

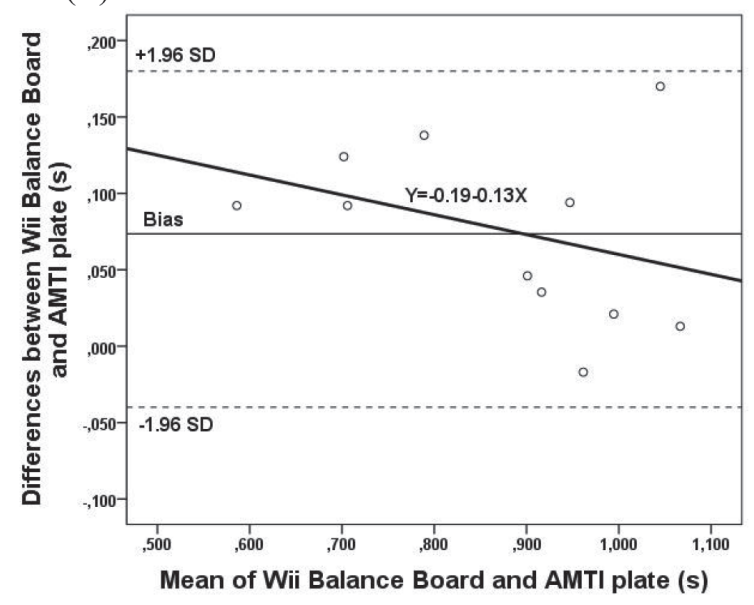

(D)

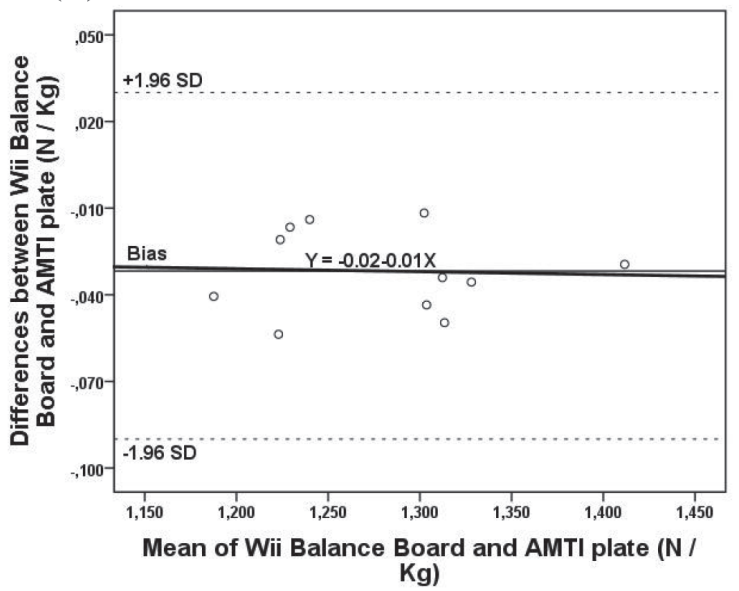

(F)

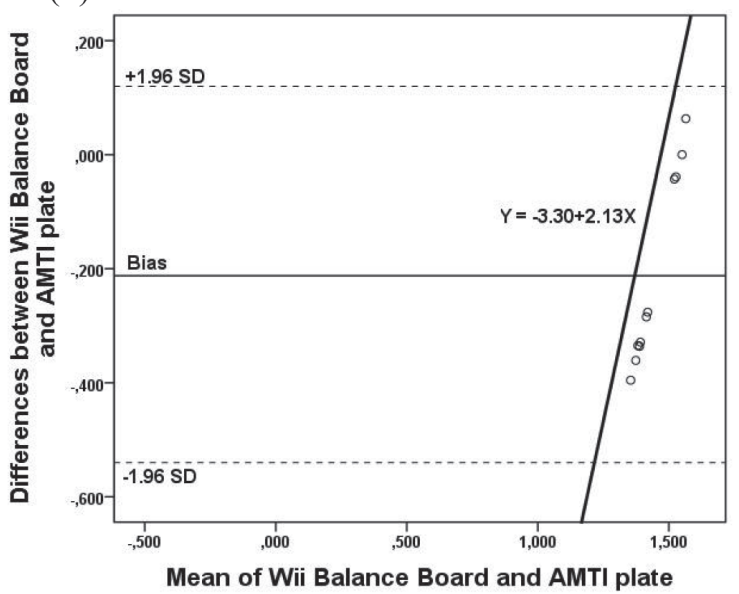

Figure 5a. Bland-Altman plots representing comparisons between the Wii Balance Board (WBB) and the laboratory-grade force platform (AMTI) for the parameters related to the STS task in the young group: (A) preparation phase; (B) rising phase; (C) stabilization phase; (D) normalized max vGRF; (E) overshoot; $(F)$ inclination of $v G R F$ in STS. The mean line represents the mean difference between the devices, with the upper and lower lines representing the limits of agreement ( $\pm 1.96 * S D)$. Solid bold lines represent the ordinary least square regression of differences on mean, with the indication of the relative equation. Note. STS: sit-to-stand; SD: standard deviation; max vGRF: maximum value of the vertical component of ground reaction force. 
(G)

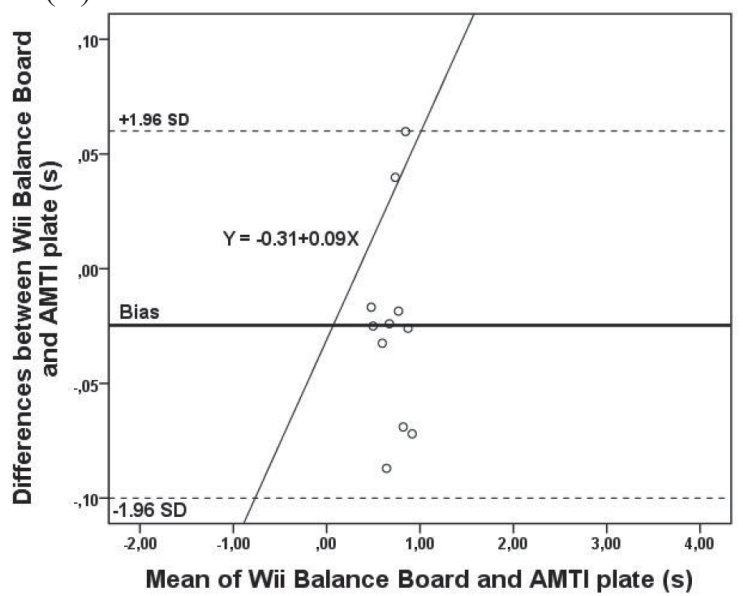

(I)

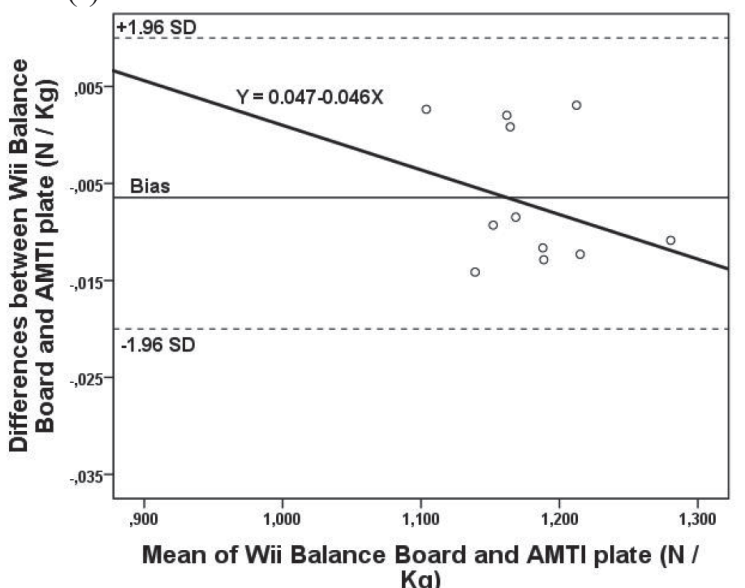

(H)

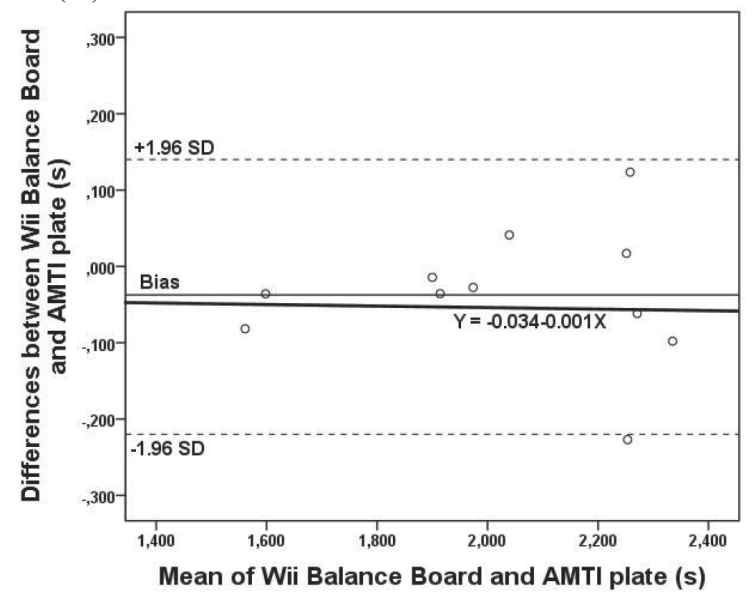

(L)

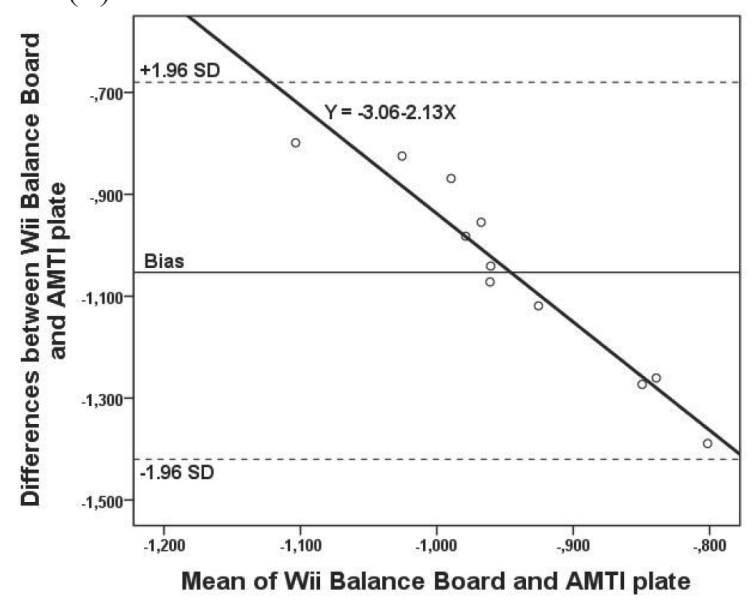

Figure 5b. Bland-Altman plots representing comparisons between the Wii Balance Board (WBB) and the laboratory-grade force platform (AMTI) for the parameters related to the RTS task in the young group: (G) preparation phase; (H) sitting phase; (I) normalized eccentric force; $(L)$ inclination of $v G R F$ in RTS. The mean line represents the mean difference between the devices, with the upper and lower lines representing the limits of agreement $\left( \pm 1.96^{*} S D\right)$. Solid bold lines represent the ordinary least square regression of differences on mean, with the indication of the relative equation. Note. RTS: return-to-sit; SD: standard deviation; $v G R F$ : vertical component of ground reaction force.

(A)

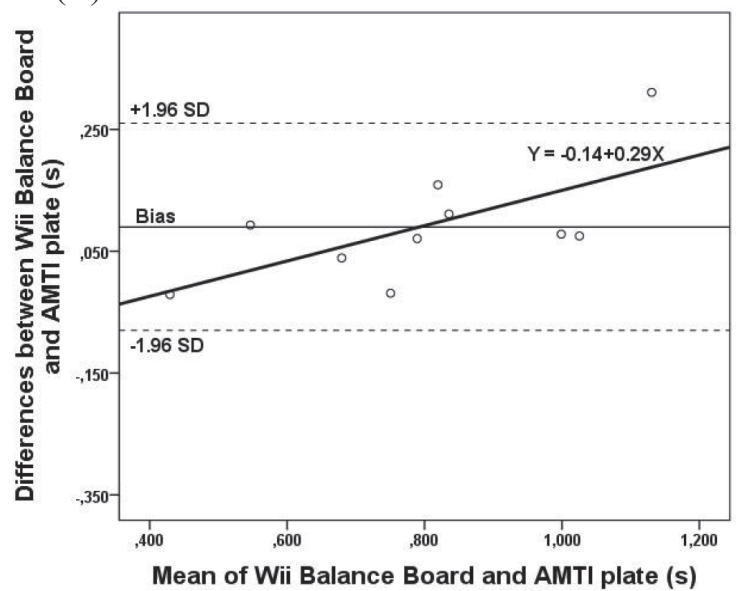

(B)

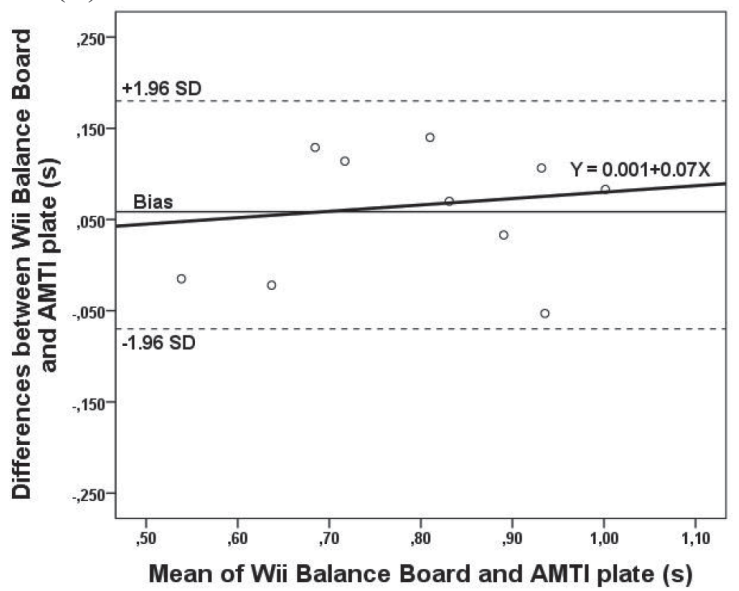


(C)

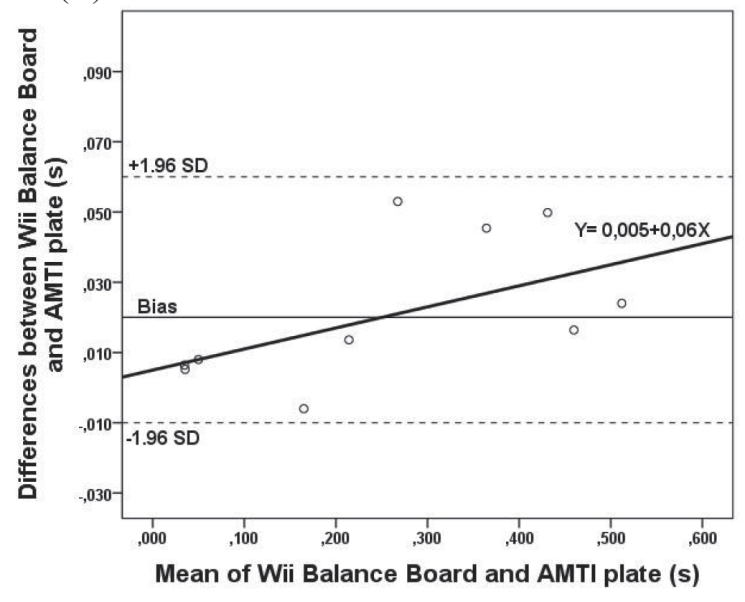

(E)

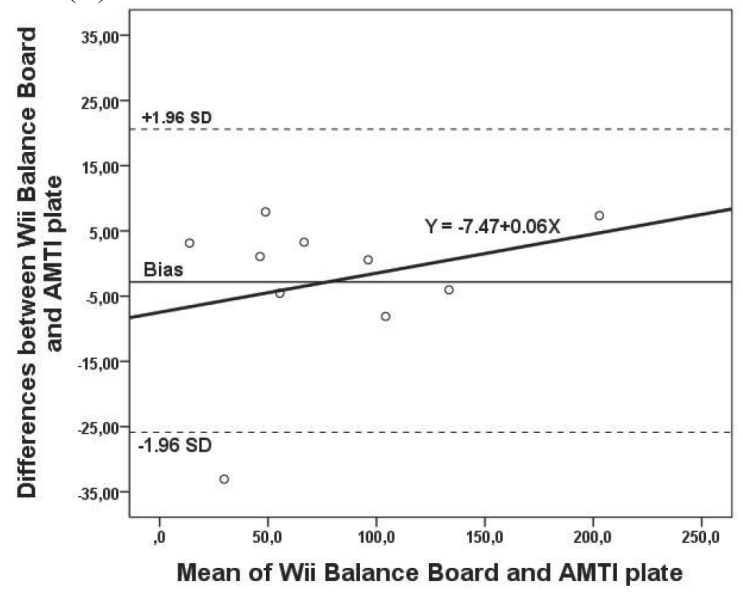

(D)

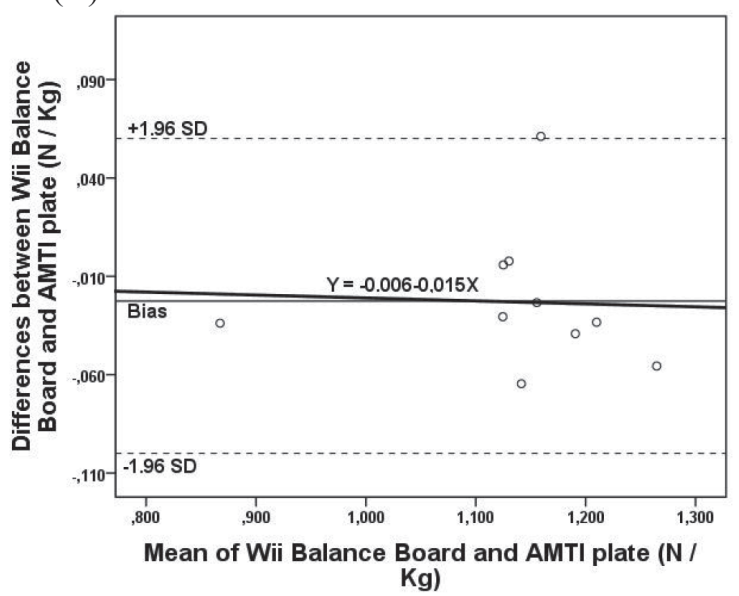

(F)

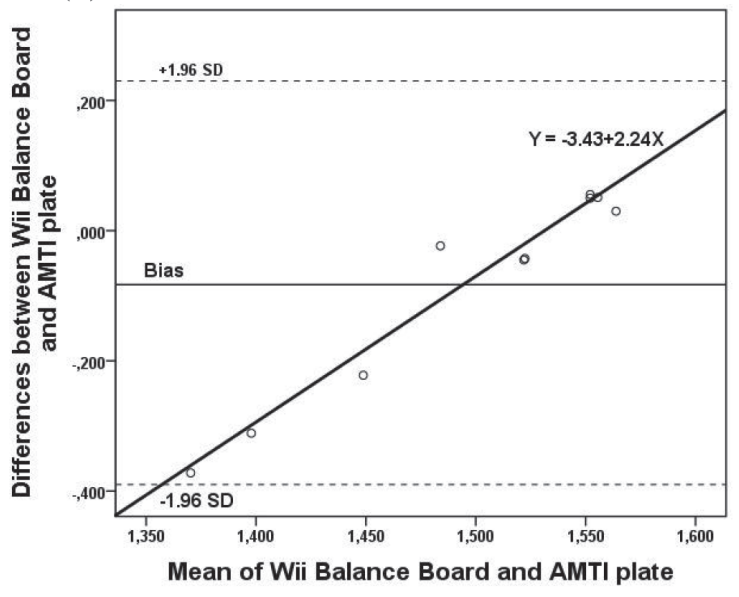

Figure 6a. Bland-Altman plots representing comparisons between the Wii Balance Board (WBB) and the laboratory-grade force platform (AMTI) for the parameters related to the STS task in the elderly group: (A) preparation phase; (B) rising phase; (C) stabilization phase; (D) normalized max $v G R F ;(E)$ overshoot; $(F)$ inclination of $v G R F$ in STS. The mean line represents the mean difference between the devices, with the upper and lower lines representing the limits of agreement ( $\pm 1.96 * S D)$. Solid bold lines represent the ordinary least square regression of differences on mean, with the indication of the relative equation. Note. STS: sit-to-stand; SD: standard deviation; max vGRF: maximum value of the vertical component of ground reaction force.

(G)

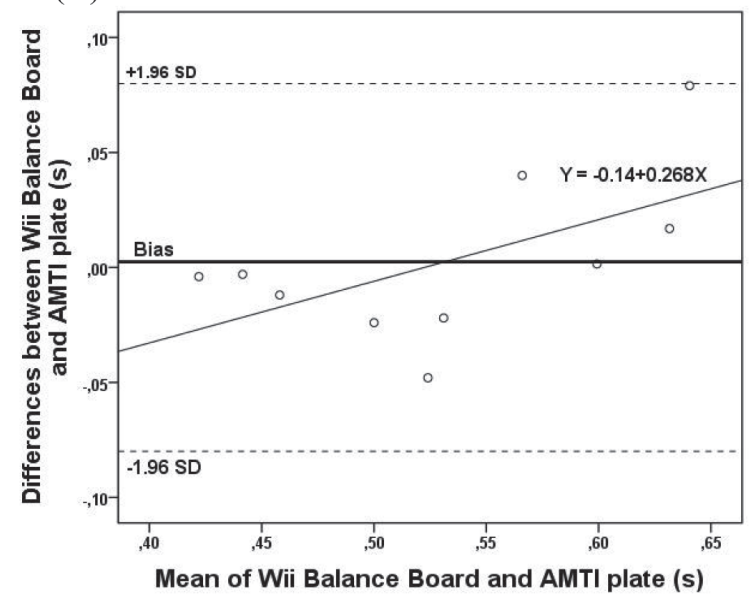

(H)

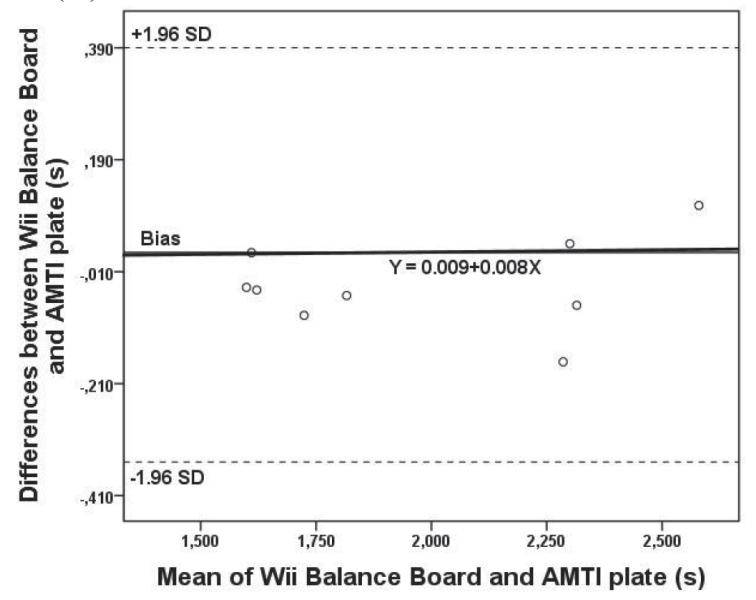


(I)

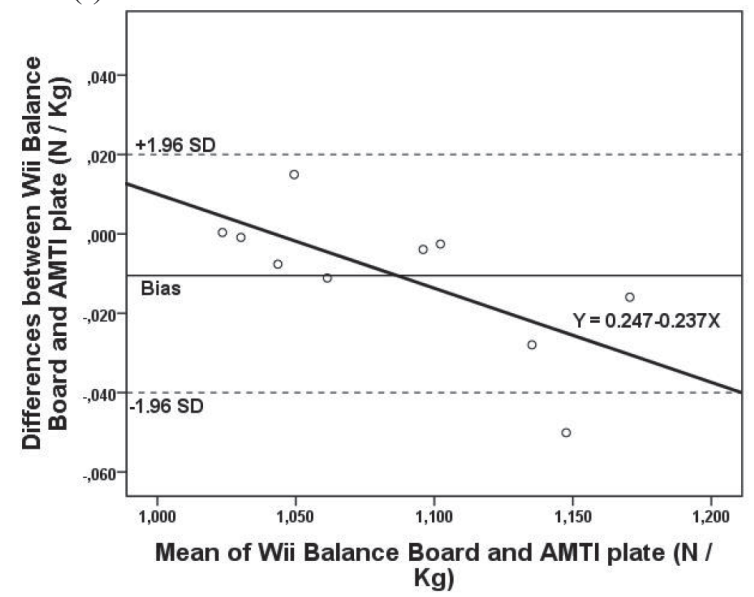

(L)

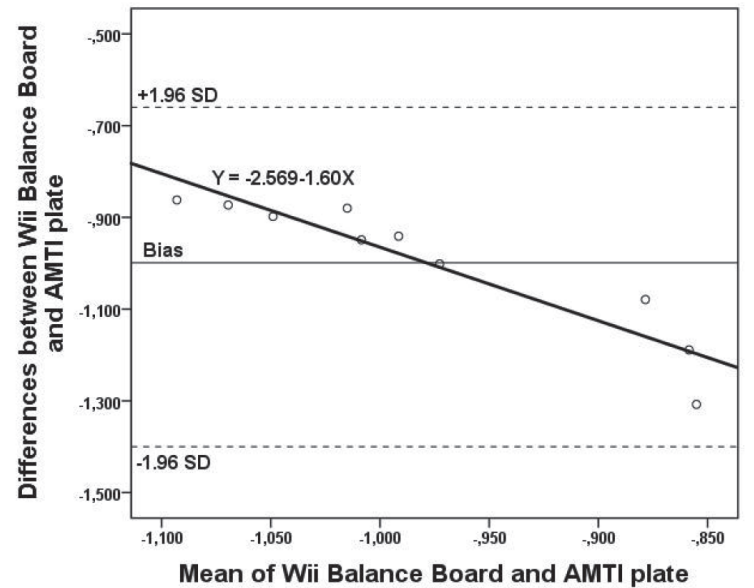

Figure 6b. Bland-Altman plots representing comparisons between the Wii Balance Board (WBB) and the laboratory-grade force platform (AMTI) for the parameters related to the RTS task in the elderly group: (G) preparation phase; (H) sitting phase; (I) normalized eccentric force; $(L)$ inclination of $v G R F$ in RTS. The mean line represents the mean difference between the devices, with the upper and lower lines representing the limits of agreement $\left( \pm 1.96^{*} S D\right)$. Solid bold lines represent the ordinary least square regression of differences on mean, with the indication of the relative equation. Note. RTS: return-to-sit; SD: standard deviation; $v G R F$ : vertical component of ground reaction force.

Table 4. The intra-device reliability analysis for the Wii Balance Board in the assessment of sit-to-stand and return-to-sit tasks for the young and the elderly

\begin{tabular}{|c|c|c|c|c|c|}
\hline Parameter & Group & M & SD & $\mathrm{ICC}(90 \% \mathrm{Cl})$ & SEM \\
\hline \multirow{2}{*}{$\mathrm{P} 1[\mathrm{~s}]$} & Young & 0.76 & 0.39 & $0.90^{* * *}(0.66,0.97)$ & 0.12 \\
\hline & Elderly & 0.82 & 0.43 & $0.93^{* * *}(0.73,0.98)$ & 0.11 \\
\hline \multirow{2}{*}{$\mathrm{P} 2[\mathrm{~s}]$} & Young & 0.92 & 0.43 & $0.86^{* * *}(0.57,0.96)$ & 0.16 \\
\hline & Elderly & 0.81 & 0.43 & $0.95^{* * *}(0.79,0.99)$ & 0.09 \\
\hline \multirow{2}{*}{ P3 [s] } & Young & 0.22 & 0.21 & $0.97^{* * *}(0.88,0.99)$ & 0.04 \\
\hline & Elderly & 0.24 & 0.23 & $0.99^{* * *}(0.97,0.99)$ & 0.02 \\
\hline \multirow{2}{*}{$\max \_v G R F_{N}[N / k g]$} & Young & 1.26 & 0.50 & $0.91^{* * *}(0.71,0.98)$ & 0.15 \\
\hline & Elderly & 1.14 & 0.50 & $0.87^{* * *}(0.53,0.97)$ & 0.18 \\
\hline \multirow{2}{*}{$\mathrm{OS}[\mathrm{N}]$} & Young & 173.2 & 5.88 & $0.89^{* * *}(0.64,0.97)$ & 1.95 \\
\hline & Elderly & 81.1 & 4.24 & $0.80^{* * *}(0.34,0.95)$ & 1.89 \\
\hline \multirow{2}{*}{ IC_Stand } & Young & 1.34 & 0.52 & $0.97^{* * *}(0.88,0.99)$ & 0.09 \\
\hline & Elderly & 1.44 & 0.57 & $0.91^{* * *}(0.68,0.98)$ & 0.17 \\
\hline \multirow{2}{*}{$\mathrm{P} 4[\mathrm{~s}]$} & Young & 0.70 & 0.37 & $0.99^{* * *}(0.97,0.99)$ & 0.04 \\
\hline & Elderly & 0.53 & 0.35 & $0.85^{\star * *}(0.46,0.96)$ & 0.13 \\
\hline \multirow{2}{*}{ P5 [s] } & Young & 1.99 & 0.63 & $0.95^{\star * *}(0.83,0.99)$ & 0.14 \\
\hline & Elderly & 1.98 & 0.66 & $0.99^{* * *}(0.97,0.99)$ & 0.07 \\
\hline \multirow{2}{*}{$\mathrm{EF}_{\mathrm{N}}[\mathrm{N} / \mathrm{kg}]$} & Young & 1.79 & 0.49 & $0.95^{\star * *}(0.81,0.99)$ & 0.11 \\
\hline & Elderly & 1.08 & 0.49 & $0.99^{* * *}(0.90,0.99)$ & 0.05 \\
\hline \multirow{2}{*}{ IC_Sit } & Young & -1.47 & 0.54 & $0.84^{\star * *}(0.52,0.96)$ & 0.22 \\
\hline & Elderly & -1.48 & 0.57 & $0.79^{* * *}(0.32,0.95)$ & 0.26 \\
\hline
\end{tabular}

Note. M: mean; SD: standard deviation; ICC: intra-class correlation coefficient; $90 \% \mathrm{Cl}$ : $90 \%$ confidence interval; SEM: standard error of measurements; P1: preparation phase; P2: rising phase; P3: stabilization phase; max_vGRF $_{\mathrm{N}}$ : the maximum value of the vertical component of ground reaction force (VGRF) normalized by the body mass; OS: overshoot; IC_Stand: slope of the vGRF during the standing phase; P4: preparation phase; P5: sitting phase; $\mathrm{EF}_{\mathrm{N}}$ : eccentric force measured as the maximum value of the VGRF normalized by the body mass; IC_Sit: slope of the VGRF during the sitting phase; ${ }^{* * *}: p<0.001$. 
for studying lower-limb weight-bearing asymmetry in clinical populations (Clark, et al., 2014), the use of a standalone WBB may represent a practical and easy-to-use solution for assessing kinetic and temporal characteristics of transitional movements because it requires calibration, synchronization and data management procedures less complex than the ones required by using two WBBs simultaneously. Therefore, the current analysis and results may be useful to support its use.

The WBB was valid and reliable for the measurement of almost all the models' parameters used for examining the pattern of movements required by STS and RTS. Concurrent validity analysis showed an excellent correlation between the measures obtained by the WBB versus those from the AMTI, and the bootstrapping analysis calculated very narrowed CIs for all the parameters with the statistically significant Pearson's product-moment correlation coefficient. Moreover, the slope of the regression line was close or very close to the identity line (i.e., slope $=1$ ) for several of the aforementioned significant parameters (i.e., P2, P3, max $\mathrm{vGRF}_{\mathrm{N}}, \mathrm{OS}, \mathrm{P} 5$, and $\mathrm{EF}_{\mathrm{N}}$ ). It means that the two devices acquired very similar values. These results suggest a high level of agreement between the two plates, meaning that the WBB could be considered a valid low-cost device for the analysis of the patterns of movement used in the afore-mentioned tasks. Consequently, our first hypothesis is verified. However, the level of accuracy of the WBB acquisitions is somewhat affected by the presence of either the proportional or fixed biases. The data shown in Table 3 revealed that only the parameters related to the inclination (i.e., IC Stand and IC Sit) were affected by the proportional bias. However, these parameters had very low validity throughout the current analyses. A possible explanation of these results could be linked to the evidence provided by Pagnacco and colleagues (2010) about the noise related to the WBB's low sample rate. Anyway, these indexes could provide interesting information about the pattern of movements performed in the afore-mentioned tasks, therefore the use of different filtering procedures or resampling approaches should be the aim of future studies. As regards the other parameters, none were affected by the proportional bias. This result assured us to detect the fixed bias with a high level of accuracy (Ludbrook, 2002). The fixed bias was detected for the temporal phases related to the standing movement in both groups: WBB data tended to be slightly higher than those measured by the AMTI. These differences might be explained by considering that the AMTI frame rate is 20 times higher than the one of the WBB, since the phases calculated from AMTI signals are slightly lower. Similar evidences were found by previous validity analysis of other devices used for assessing human movement (Huurnink, et al.,
2013). Pagnacco and colleagues (2010) argued that a low sampling rate of the WBB might produce an increment of the noise and it might contribute to the biases between the two devices. Of note, the magnitude of those biases was small for P1 and P2, but trivial for P3. Moreover, the analysis of the regression line between the data points on the Bland-Altman plots revealed slopes very close to the 0 for all those parameters: these results mean that the differences between WBB and AMTI were similar across the whole range of values analysed in each phase. The stabilization phase showed the highest level of accuracy both in the elderly and in the young, while the preparation and rising phases showed the lowest level of accuracy. Considering the return-to-sit transition, the biases of the temporal phases were more limited than those of the STS temporal phases, and were interpreted as trivial. The fixed biases were detected for the kinetic parameters (i.e., $\max \mathrm{vGRF}_{\mathrm{N}}$ and $\mathrm{EF}_{\mathrm{N}}$ ) related to the STS and RTS in the young group. Potential reasons for those biases include hardware configuration (Pagnacco, et al., 2010), anthropometric differences between subjects (i.e., the young had mean weight higher than the elderly) (Sañudo, et al., 2016), and movements with possible peaks of excessive shock (Yamamoto \& Matsuzawa, 2013). Therefore, the WBB seems to provide a higher level of accuracy on the measures related to the phases of the tasks characterized by low movement dynamics and measurements not dependent on the participants' age. Accordingly, the use of WBB seem to be adequate for practitioners who have the need to assess low dynamic motion as well as to detect deterioration in elderly's motor performance required in activities of daily living. If the aim is related to moderate-to-high performance, the use of wii balance board seem to be adequate when a low level of accuracy in kinetic parameters assessment is required.

The ICC's results for intra-device reliability showed excellent levels for all parameters and they support our second hypothesis. The reliability analysis did not show appreciable differences for any of the statistics between the sampled groups. The limited values of the standard error of measurement confirmed the test-retest validity of the WBB and were in accordance with previous results (Clark, et al., 2010; Jorgensen, et al., 2015). The largest differences among temporal parameters were in the rising (P3) and sitting (P5) phases, which were related to the pattern of movements characterized by the highest level of dynamics, this characteristic apparently being the main drawback of the accuracy and reliability of the WBB. The reliability statistics for explosive force $\left(\max \_\mathrm{vGRF}_{\mathrm{N}}\right)$ were 0.91 and 0.87 for the young and the elderly, respectively, and they were lower than the values obtained for normalized eccentric force $\left(\mathrm{EF}_{\mathrm{N}}\right)(0.95$ and 0.99 for the 
young and the elderly, respectively). This could mean that the explosive force produced during the standing phase is less reliable than the eccentric force measured during the sitting phase. Indeed, according to a previous study (Van Lummel, et al., 2013), the explosive force seems to be a less stable kinetic parameter during these transitional movements than eccentric force and this could be considered a possible explanation for the current evidence. Finally, this difference could be considered an implication of the mechanical consideration provided by Yamamoto and Matsuzawa (2013) about the moderate level of linearity provided by the WBB if high peak forces were applied on its surface.

The generalizability of the current results is limited by the following issues: a) the sample size is relatively small, while better results may be obtained by involving a large number of participants; $b$ ) the current analyses need to be further investigated by considering also patients with locomotor dysfunctions (e.g., subjects with anterior-lateral ligament reconstruction) with the aim to provide worthwhile indications for using a standalone WBB also with clinical populations.

In conclusion, the current results seem to suggest that the WBB is valid and reliable device to assess the pattern of movements required in STS and RTS, but the current analysis confirmed the need to pay attention for using WBB and force plates interchangeably. However, the reduced accuracy of WBB compared to the AMTI is limited and can be balanced out by the following WBB characteristics: low-cost, easy-to-use, and portable. Hence, this study is relevant for practitioners involved in the ecological assessment of human movement, such as therapists, physical education teachers, and performance analysts. Finally, these evidences can also represent the groundwork for the development of rehabilitation exergames in which the WBB signal could be used for the accurate motion animation of an avatar who facilitates improvements of the participants' experience of re-learning movement (Bonnechère, 2017; Zeng, Pope, Lee, \& Gao, 2017).

\section{References}

Abujaber, S., Gillispie, G., Marmon, A., \& Zeni, J. (2015). Validity of the Nintendo Wii Balance Board to assess weight bearing asymmetry during sit-to-stand and return-to-sit task. Gait \& Posture, 41(2), 676-682.

Bonnechère, B. (2017). Serious games in physical rehabilitation: From theory to practice. Springer.

Clark, R.A., Bryant, A.L., Pua, Y., McCrory, P., Bennell, K., \& Hunt, M. (2010). Validity and reliability of the Nintendo Wii Balance Board for assessment of standing balance. Gait \& Posture, 31(3), 307-310.

Clark, R.A., Howells, B., Feller, J., Whitehead, T., \& Webster, K.E. (2014). Clinic-based assessment of weight-bearing asymmetry during squatting in people with anterior cruciate ligament reconstruction using Nintendo Wii Balance Boards. Archives of Physical Medicine and Rehabilitation, 95(6), 1156-1161.

Clark, R.A., Mentiplay, B.F., Pua, Y.H., \& Bower, K.J. (2018). Reliability and validity of the Wii Balance Board for assessment of standing balance: A systematic review. Gait \& Posture, 61, 40-54.

Davis, W.E., \& Burton, A.W. (1991). Ecological task analysis: Translating movement behavior theory into practice. Adapted Physical Activity Quarterly, 8(2), 154-177.

Fleiss, J.L. (2011). Design and analysis of clinical experiments. New York: John Wiley \& Sons.

Giavarina, D. (2015). Understanding Bland Altman analysis. Biochemia Medica, 25(2), 141-151.

Guralnik, J.M., Simonsick, E.M., Ferrucci, L., Glynn, R.J., Berkman, L.F., Blazer, D.G., ... \& Wallace, R.B. (1994). A short physical performance battery assessing lower extremity function: Association with self-reported disability and prediction of mortality and nursing home admission. Journal of Gerontology, 49(2), M85-M94.

Hopkins, W. (2000). Analysis of validity by linear regression. Retrieved November 30, 2016 from: http://sportsci.org/ resource/stats/xvalid.xls

Hortobágyi, T., Zheng, D., Weidner, M., Lambert, N.J., Westbrook, S., \& Houmard, J.A. (1995). The influence of aging on muscle strength and muscle fiber characteristics with special reference to eccentric strength. The Journals of Gerontology Series A: Biological Sciences and Medical Sciences, 50(6), B399-B406.

Huurnink, A., Fransz, D.P., Kingma, I., \& van Dieën, J.H. (2013). Comparison of a laboratory grade force platform with a Nintendo Wii Balance Board on measurement of postural control in single-leg stance balance tasks. Journal of Biomechanics, 46(7), 1392-1395.

Janssen, W.G., Bussmann, H.B., \& Stam, H.J. (2002). Determinants of the sit-to-stand movement: A review. Physical Therapy, 82(9), 866-879.

Jorgensen, M.G., Andersen, S., Ryg, J., \& Masud, T. (2015). Novel use of the Nintendo Wii Board for measuring isometric lower limb strength: A reproducible and valid method in older adults. PLoS One, 10(10).

Kralj, A., Jaeger, R.J., \& Munih, M. (1990). Analysis of standing up and sitting down in humans: Definitions and normative data presentation. Journal of Biomechanics, 23(11), 1123-1138.

Lindemann, U., Claus, H., Stuber, M., Augat, P., Muche, R., Nikolaus, T., \& Becker, C. (2003). Measuring power during the sit-to-stand transfer. European Journal of Applied Physiology, 89(5), 466-470. 
Ludbrook, J. (2002). Statistical techniques for comparing measurers and methods of measurement: A critical review. Clinical and Experimental Pharmacology and Physiology, 29(7), 527-536.

Mengarelli, A., Verdini, F., Cardarelli, S., Di Nardo, F., Burattini, L., \& Fioretti, S. (2018). Balance assessment during squatting exercise: A comparison between laboratory grade force plate and a commercial, low-cost device. Journal of Biomechanics, 71, 264-270.

Merchant-Borna, K., Jones, C.M.C., Janigro, M., Wasserman, E.B., Clark, R.A., \& Bazarian, J.J. (2017). Evaluation of Nintendo Wii balance board as a tool for measuring postural stability after sport-related concussion. Journal of Athletic Training, 52(3), 245-255.

Pagnacco, G., Oggero, E., \& Wright, C.H.G. (2010). Biomedical instruments versus toys: A preliminary comparison of force platforms and the Nintendo Wii Balance Board. Biomedical Sciences Instrumentation, 47, 12-17.

Papa, E., \& Cappozzo, A. (2000). Sit-to-stand motor strategies investigated in able-bodied young and elderly subjects. Journal of Biomechanics, 33(9), 1113-1122.

Park, D.S., \& Lee, G. (2014). Validity and reliability of balance assessment software using the Nintendo Wii balance board: Usability and validation. Journal of NeuroEngineering and Rehabilitation, 11, 99.

Pavan, P., Cardaioli, M., Ferri, I., Gobbi, E., \& Carraro, A. (2015). A contribution to the validation of the Wii Balance Board for the assessment of standing balance. European Journal of Sport Science, 15(7), 600-605.

Roig, M., MacIntyre, D.L., Eng, J.J., Narici, M.V., Maganaris, C.N., \& Reid, W.D. (2010). Preservation of eccentric strength in older adults: Evidence, mechanisms and implications for training and rehabilitation. Experimental Gerontology, 45(6), 400-409.

Sañudo, B., Rueda, D., del Pozo-Cruz, B., de Hoyo, M., \& Carrasco, L. (2016). Validation of a video analysis software package for quantifying movement velocity in resistance exercises. Journal of Strength and Conditioning Research, 30(10), 2934-2941.

Sgrò, F., Licari, D., Coppola, R., \& Lipoma, M. (2015). Assessment of balance abilities in elderly people by means of a clinical test and a low-cost force plate. Kinesiology, 47(1), 33-43.

Sgrò, F., Monteleone, G., Pavone, M., \& Lipoma, M. (2014). Validity analysis of Wii Balance Board versus baropodometer platform using an open custom integrated application. AASRI Procedia, 8, 22-29.

Silva, P.F., Quintino, L.F., Franco, J., \& Faria, C.D. (2014). Measurement properties and feasibility of clinical tests to assess sit-to-stand/stand-to-sit tasks in subjects with neurological disease: A systematic review. Brazilian Journal of Physical Therapy, 18(2), 99-110.

Van Lummel, R.C., Ainsworth, E., Lindemann, U., Zijlstra, W., Chiari, L., Van Campen, P., \& Hausdorff, J.M. (2013). Automated approach for quantifying the repeated sit-to-stand using one body fixed sensor in young and older adults. Gait \& Posture, 38(1), 153-156.

Whitney, S.L., Wrisley, D.M., Marchetti, G.F., Gee, M.A., Redfern, M.S., \& Furman, J.M. (2005). Clinical measurement of sit-to-stand performance in people with balance disorders: Validity of data for the Five-Times-Sit-to-Stand Test. Physical Therapy, 85(10), 1034-1045.

Winter, D.A. (1995). Human balance and posture control during standing and walking. Gait \& Posture, 3(4), $193-214$.

Yamako, G., Chosa, E., Totoribe, K., Fukao, Y., \& Deng, G. (2017). Quantification of the sit-to-stand movement for monitoring age-related motor deterioration using the Nintendo Wii Balance Board. PLoS one, 12(11), e0188165.

Yamamoto, K., \& Matsuzawa, M. (2013). Validity of a jump training apparatus using Wii Balance Board. Gait \& Posture, 38(1), 132-135.

Zeng, N., Pope, Z., Lee, J.E., \& Gao, Z. (2017). A systematic review of active video games on rehabilitative outcomes among older patients. Journal of Sport and Health Science, 6(1), 33-43.

Zijlstra, A., Mancini, M., Lindemann, U., Chiari, L., \& Zijlstra, W. (2012). Sit-stand and stand-sit transitions in older adults and patients with Parkinson's disease: Event detection based on motion sensors versus force plates. Journal of NeuroEngineering and Rehabilitation, 9, 75.

Submitted: April 5, 2017

Accepted: April 13, 2018

Published Online First: January 15, 2019

Correspondence to:

Francesco Sgrò, Ph.D.

Faculty of Human and Society Sciences - University

of Enna "Kore"

Cittadella Universitaria, 94100, Enna, EN, Italy

Phone: +39 3495863425

Fax: +39 0935536993

E-mail: francesco.sgro@unikore.it 